\title{
Technè
}

La science au service de l'histoire de l'art et de la préservation des biens culturels

$45 \mid 2017$

Bronzes grecs et romains : études récentes sur la statuaire antique

\section{Les yeux d'Auguste et de Livie au musée du Louvre : une nouvelle technique antique identifiée}

The eyes of Augustus and Livia at the Louvre: a new Antique technique identified

Sophie Descamps-Lequime, Isabelle Biron et Juliette Langlois

\section{OpenEdition}

Journals

Édition électronique

URL : http://journals.openedition.org/techne/1304

DOI : 10.4000/techne.1304

ISSN : 2534-5168

Éditeur

C2RMF

Édition imprimée

Date de publication : 1 mai 2017

Pagination : 84-99

ISBN : 978-2-7118-6408-9

ISSN : $1254-7867$

\section{Référence électronique}

Sophie Descamps-Lequime, Isabelle Biron et Juliette Langlois, « Les yeux d'Auguste et de Livie au musée du Louvre : une nouvelle technique antique identifiée », Technè [En ligne], 45 | 2017, mis en ligne le 19 décembre 2019, consulté le 22 juillet 2020. URL : http://journals.openedition.org/techne/1304 ; DOI : https://doi.org/10.4000/techne.1304

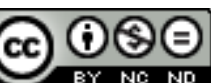

La revue Technè. La science au service de l'histoire de l'art et de la préservation des biens culturels est mise à disposition selon les termes de la Licence Creative Commons Attribution - Pas d'Utilisation Commerciale - Pas de Modification 4.0 International. 


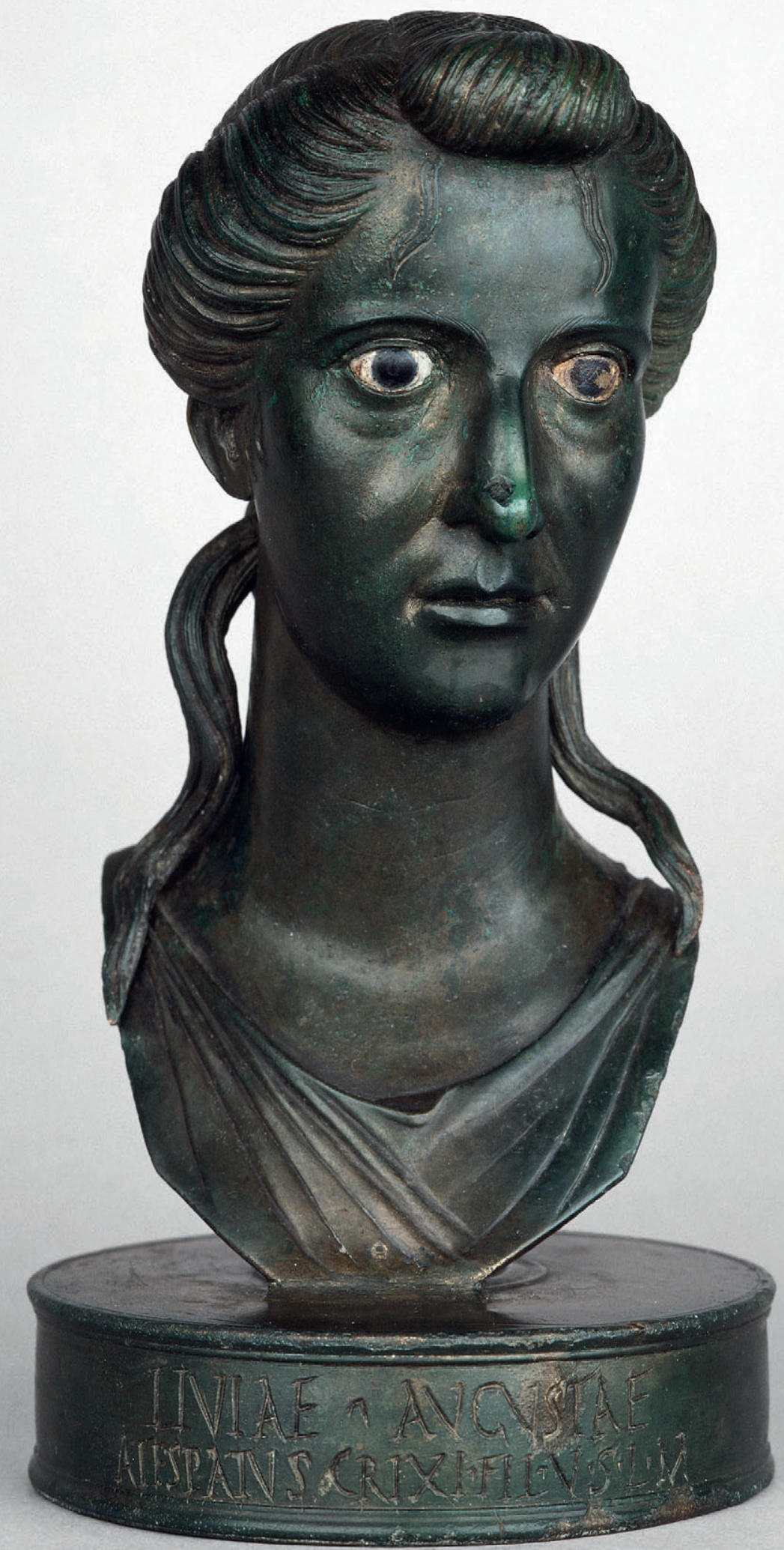

Fig. 1. Portrait de Livie, Neuilly-le-Réal (Allier), Paris, musée du Louvre, département des Antiquités grecques, étrusques et romaines, $\mathrm{Br} 28$. @ Musée du Louvre et AFA/D. Lebée et C. Déambrosis. 
Sophie Descamps-Lequime

Isabelle Biron

Juliette Langlois

Monique Dondin-Payre

\section{Les yeux d'Auguste et de Livie au musée du Louvre : une nouvelle technique antique identifiée}

The eyes of Augustus and Livia at the Louvre: a new Antique technique identified

Résumé. La collaboration entre le musée du Louvre et le C2RMF a permis de réhabiliter définitivement les portraits en bronze d'Auguste et de Livie, découverts à Neuilly-le-Réal (France, Allier) en 1816. L'étude a démontré que les anomalies à la fois typologiques, stylistiques et épigraphiques, constatées à plusieurs reprises, devaient être interprétées comme liées au contexte gallo-romain de création des deux bustes. Une nouvelle technique antique de fabrication des yeux (verres blancs opaques, verres d'aspect noir, sans césure entre les différentes couleurs) a été mise en évidence. Les analyses chimiques élémentaires confirment l'appartenance des yeux à la production verrière de luxe - vaisselle colorée décorée de camées, verres millefiori, bijoux -, probablement entre 30 et 70. Il est dès lors proposé de dater les deux ex-voto, consacrés par Atespatus, après la mort d'Auguste.

Mots-clés. Portrait, Auguste, Livie, Atespatus, bronze, yeux, verre, adhésif, romain impérial, Neuilly-le-Réal.
Abstract. Discovered in Neuilly-le-Réal (Allier, France) in 1816, the bronze portraits of Augustus and Livia have been decisively rehabilitated as a result of a collaborative project undertaken by the Louvre and the C2RMF. Research has shown that the typological, stylistic and epigraphic anomalies, observed on several occasions, should be related to the Gallo-Roman context of the creation of the two busts. An unprecedented ancient technique in the manufacture of the eyes (opaque white glass, black-looking glass with no division between the different colours) came to light. Elementary chemical analysis confirmed that the eyes were produced using luxury glassmaking techniques - coloured glassware with cameo decoration, millefiori glassware, jewellery probably between $A D 30$ and $A D$ 70. The two ex-votos, dedicated by Atespatus, may thus be dated to after the death of Augustus.

Keywords. Portrait, Augustus, Livia, Atespatus, bronze, eyes, glass, adhesive, Roman imperial period, Neuilly-le-Réal.

\section{Une authenticité discutée}

Depuis leur exhumation fortuite le 7 mai 1816 et leur acquisition par le musée du Louvre en 1868 grâce à l'implication de Prosper Mérimée, qui n'avait pas hésité à se rendre à Londres alors qu'ils étaient sur le point d'être acquis par le British Museum, les deux petits portraits en bronze d'Auguste et de Livie ont divisé les spécialistes ${ }^{1}$. Peu d'œuvres ont suscité autant d'interrogations, certains auteurs les jugeant antiques et d'autres les considérant comme des faux à cause des conditions de leur découverte, de leur remarquable état de conservation et d'anomalies à la fois typologiques, stylistiques et épigraphiques (fig. 1-2). Les doutes émis à plusieurs reprises ont conduit à les maintenir en réserve durant de nombreuses décennies. Mais l'étude récente, menée au C2RMF dans le cadre de leur restauration, a permis de les réhabiliter ${ }^{2}$.

\section{Circonstances de la découverte}

Les deux bustes, hauts respectivement de 18,5 et $19 \mathrm{~cm}$, ont été mis au jour à Neuilly-le-Réal par deux jeunes laboureurs, qui retournaient la terre d'un champ près d'une rivière ${ }^{3}$. Les bronzes gisaient à proximité l'un de l'autre, à une faible profondeur. Un tel contexte pouvait faire craindre une mise en scène destinée à abuser les contemporains de la trouvaille, à la manière de celle qui avait pu tromper le comte de Caylus en 1763 lors de la découverte à Chalon-sur-Saône d'un coffret contenant dix-huit statuettes de bronze, parmi lesquelles des œuvres antiques et modernes ${ }^{4}$. Il incitait à la prudence, d'autant plus que le lieu d'exhumation des bustes, le « Domaine des Guillemins », repérable sur le cadastre napoléonien, ne devait livrer par la suite aucun autre vestige antique.

Les circonstances de la découverte sont en effet connues. Elles ont été très précisément consignées par le notaire qui procéda à la vente des bustes le 29 mai $1816^{5}$. Le procès-verbal décrit l'apparence des bronzes qui présentaient un état de conservation remarquable, à l'exception des deux yeux 


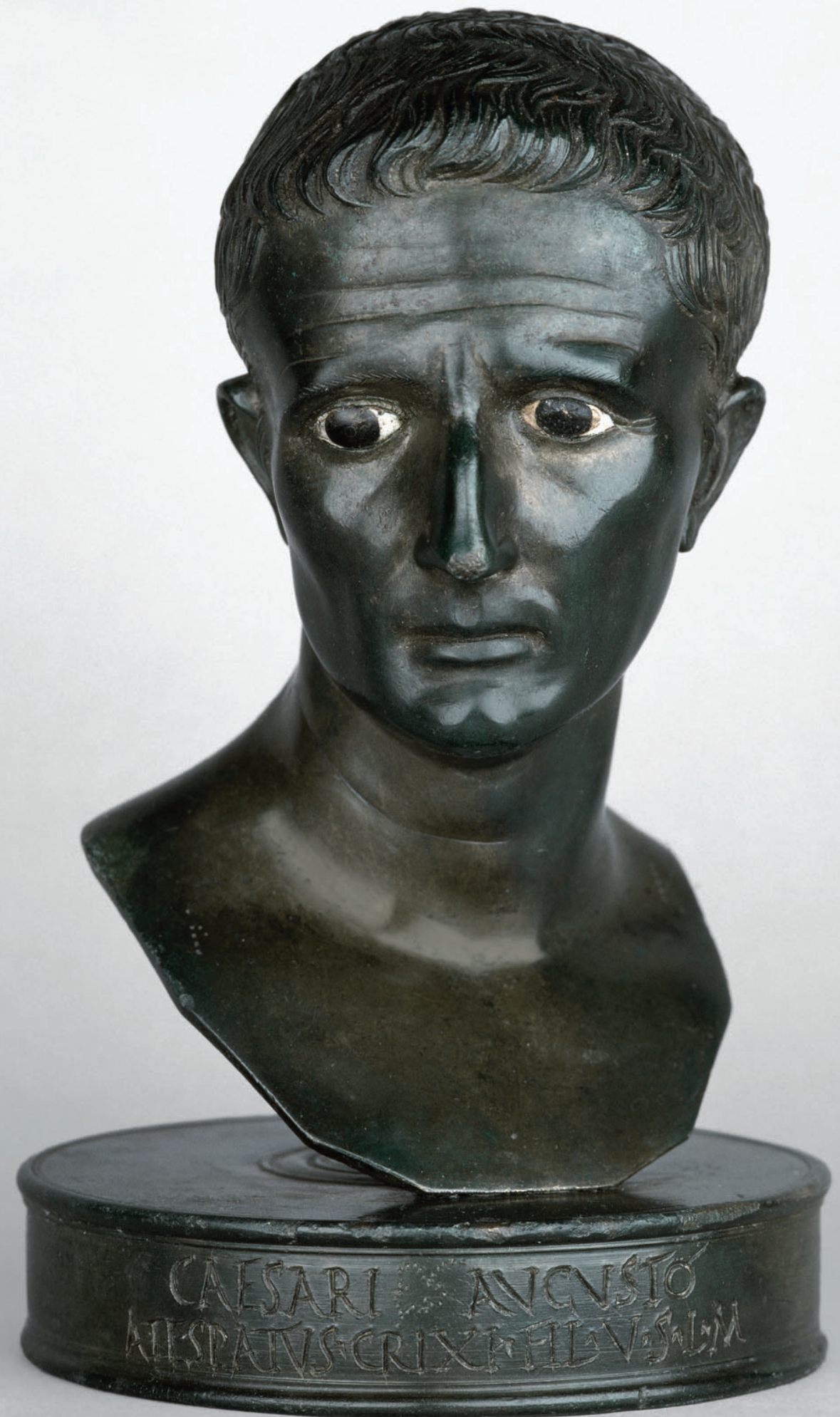

Fig. 2. Portrait d'Auguste, Neuilly-le-Réal (Allier), Paris, musée du Louvre, département des Antiquités grecques, étrusques et romaines, $\mathrm{Br} 29$. ( ) Musée du Louvre et AFA/D. Lebée et C. Déambrosis. 
gauches : «tombé par morceaux sous la main de celui qui avait lavé la tête » pour l'œil de la figure féminine et «par morceaux au moindre contact » pour celui du personnage masculin ${ }^{6}$.

\section{Anomalies}

Parmi les anomalies relevées figure la découpe polygonale des bustes, qui n'est pas documentée par ailleurs. Les portraits de petites dimensions en bronze présentent toujours un arrondi depuis le sternum jusqu'à la naissance des épaules, ce qu'illustrent par exemple un buste masculin anonyme et un portrait de femme âgée, conservés au musée du Louvre ${ }^{7}$. L'état de conservation des surfaces est également si remarquable que certains auteurs ont douté du caractère naturel de la patine vert sombre des épidermes et de sa formation au cours de siècles d'enfouissement. Les travaux en laboratoire ont montré toutefois que cette patine homogène n'était pas artificielle ${ }^{8}$. Les inscriptions votives, gravées dans la cire des modèles intermédiaires, avant la coulée, sur les parois verticales des bases circulaires, confirment quant à elles que les deux bustes formaient une paire. Elles sont cependant atypiques ${ }^{9}$.

On peut lire, pour le personnage masculin :

\section{CAESARI AVGUSTO ATESPATVS CRIXI}

FIL(ius) V(otum) S(olvit) L(ibens) M(erito)

«À César Auguste, Atespatus fils de Crixus,

a accompli son vœu de bon gré, comme il se doit. »

Et pour le personnage féminin :

\section{LIVIAE AVGVSTAE ATESPATVS CRIXI}

FIL(ius) V(otum) S(olvit) L(ibens) M(erito)

«À Livia Augusta, Atespatus fils de Crixus,

a accompli son vœu de bon gré, comme il se doit. »

Les deux bronzes ont été consacrés par Atespatus, fils de Crixus, à Auguste et Livie, en remerciement de l'exaucement d'un vœu. La divinité qui a reçu ce vœu et l'a réalisé n'est pas mentionnée. Mais les dédicaces prêtent au couple impérial des titulatures - César Auguste et « Livia Augusta » qui introduisent des incohérences chronologiques. Livie ne devint Augusta qu'à la mort d'Auguste en 14 après J.-C. L'empereur reçut alors le titre posthume de Divus : c'est donc ce titre qui aurait dû répondre à celui d'Augusta. On sait aussi que, pour respecter les dernières volontés de l'empereur qui avait spécifié dans son testament qu'il l'adoptait comme sa fille, Livie fut appelée Julie dès le début de son veuvage. Ainsi, la dénomination officielle de Livie, mère de l'empereur Tibère, fut, à partir de 14, Julia Augusta, et c'est plutôt cette titulature qui aurait dû apparaître dans l'inscription de Neuilly-le-Réal. Une nouvelle dédicace d'un Atespatus est apparue récemment sur une cruche consacrée à Mercure (voir encadré en fin d'article). Cette troisième occurrence confirme la présence du nom d'Atespatus dans la région.

\section{Des bustes qui ne s'inscrivent pas aisément dans le corpus des portraits du couple impérial}

L'impératrice est vêtue d'une tunique épaisse, qui lui couvre les épaules; le buste d'Auguste est nu. La tête de Livie est plus ronde et plus petite que celle d'Auguste ${ }^{10}$. L'artiste a volontairement insisté sur les marques de l'âge tout en adoptant une coiffure qui se réfère aux portraits juvéniles des deux empereurs. Le visage d'Auguste est émacié. La peau, tendue sur les pommettes, souligne la maigreur du modèle. L'affaissement des chairs se manifeste par la présence de zones légèrement gonflées, ainsi à proximité des ailes du nez, et de cernes sous les yeux. Les rides sont profondes. Mais la chevelure, organisée depuis le sommet du crâne en ondes régulières de mèches incurvées vers la droite puis vers la gauche, et la disposition de la courte frange évoquent les portraits de jeunesse de l'empereur (type Louvre-Forbes) ${ }^{11}$.

Une même impression de décalage se dégage de l'analyse du buste de Livie. L'aspect vieillissant du modèle est souligné par un affaissement des chairs dans la zone des joues et par des poches sous les yeux. L'impératrice porte la coiffure de sa jeunesse, dite à nodus, une coiffure à la mode dans les dernières années de l'époque républicaine ${ }^{12}$. Le nodus correspond à la protubérance formée par la chevelure, qui a été tirée vers l'avant au-dessus du front puis nattée vers l'arrière jusqu'à un chignon bas (fig. 3). Les cheveux sont relevés en bandeaux sur les tempes et au-dessus des oreilles, qu'ils

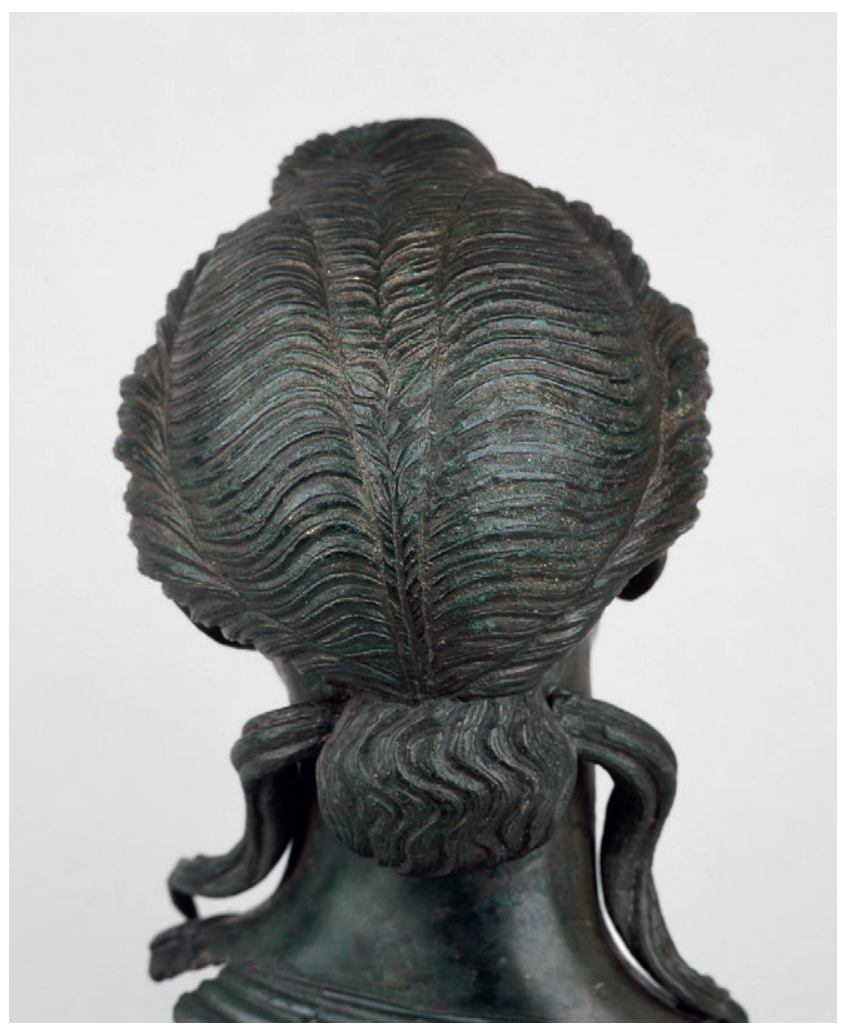

Fig. 3. Portrait de Livie, détail de la chevelure au revers. (c) Musée du Louvre et AFA/D. Lebée et C. Déambrosis. 
dissimulent en partie. Deux mèches incurvées, coulées séparément, s'échappent du chignon et retombent sur les épaules. La statue de Livie érigée à Rome en 35 avant J.-C. était coiffée du nodus (type dit de Marbury Hall ) ${ }^{13}$. Cette coiffure raffinée, dont le camée Hague est sans doute le plus bel écho, était nattée non seulement sur le sommet de la tête, mais également autour du chignon ${ }^{14}$. Le buste de Neuilly-leRéal se rapproche d'une version simplifiée de la coiffure à nodus, dont témoigne un autre type de portrait de Livie, dit du Fayoum ${ }^{15}$, sans doute créé dans les premières années de l'époque impériale. Ce second type est illustré également au Louvre par une tête en basalte de l'impératrice ${ }^{16}$. Les mèches parotides, plus rarement représentées, apparaissent sur un buste conservé à Copenhague ${ }^{17}$. On retrouve sur le bronze la forme aplatie du nodus du type du Fayoum et les petites mèches sinueuses, quoique plus longues, qui retombent sur le front, mais le traitement de la natte qui dissimule la raie médiane, les larges sillons qui parcourent la chevelure à l'arrière du crâne, l'ampleur des bandeaux au-dessus des tempes, la présence de mèches parotides et le caractère lâche du chignon sont autant d'indices d'une composition indépendante : toutes les anomalies, tous les éléments atypiques s'expliquent en fait si l'on accepte le principe d'un contexte provincial pour la commande. Dès l'an 9 avant J.-C., on observe à Rome, sur l'Ara Pacis Augustae, que l'impératrice adopte une coiffure à raie médiane $\mathrm{e}^{18}$, mais certains exemplaires des portraits à nodus révèlent que le type du Fayoum a perduré, principalement dans les lieux éloignés des grands centres producteurs.

\section{Techniques de fabrication des deux bustes : l'apport du laboratoire}

\section{Composition des bronzes et procédés de coulée}

Les analyses physico-chimiques effectuées par spectrométrie d'absorption atomique au C2RMF par Loïc Hurtel confirment que les deux bustes, ainsi que leur base, ont été coulés dans un même alliage ternaire, contenant de 5 à $6 \%$ de plomb et de 5 à $6 \%$ d'étain, selon une composition qui n'est pas incompatible avec une origine gallo-romaine ${ }^{19}$.

Les radiographies réalisées par Thierry Borel permettent de comprendre la genèse des deux portraits qui, bien que d'apparence comparable et de même alliage cuivreux, élaborés très probablement dans le même atelier, ont été réalisés de manière totalement différente. Le buste d'Auguste est, d'après ses parois métalliques minces et régulières, une fonte à la cire perdue sur négatif (fig. 4). L'artiste a pris l'empreinte d'un portrait existant, proche du type Louvre-Forbes ${ }^{20}$. Il est intervenu sur le modèle intermédiaire en cire, pour vieillir Auguste et ajouter les oreilles. Le buste de Livie est, en revanche, une fonte à la cire perdue sur positif, reconnaissable à l'épaisseur importante et irrégulière des parois (fig. 5). L'artiste a donc nécessairement travaillé directement le modèle intermédiaire, en ajoutant et en modelant les éléments de cire sur une ébauche en terre réfractaire jusqu'à obtenir le portrait souhaité. Ne disposant pas d'un modèle initial en ronde-bosse, il s'est sans doute inspiré d'une effigie bidimensionnelle de l'impératrice - un portrait monétaire ou une gemme comme la cornaline du trésor de Petescia trouvé aux environs de Rome : l'impératrice y apparaît de trois quarts, coiffée, de manière naturaliste, du nodus et de mèches parotides. Mentionnons également le camée de verre conservé à Berlin, sur lequel Livie de profil est coiffée à la manière du type du Fayoum, avec une mèche échappée du chignon $^{21}$.

\section{La fabrication des yeux}

L'étude des yeux a apporté un argument décisif en faveur de l'authenticité des bustes. Si les yeux gauches étaient très altérés au moment de la découverte et n'ont pu être conservés, il n'en allait pas de même des yeux droits. Celui de Livie est déjà décrit en 1816 comme «à peine terni ». Le caractère antique des deux yeux préservés a été confirmé par l'analyse. Mais c'est surtout une technique insoupçonnée jusqu'alors, qui a été mise en évidence à l'occasion de la récente investigation ${ }^{22}$.

Il a été possible de déchausser l'œil droit de Livie afin de l'étudier plus facilement (fig. 6-7). Cela a permis de confirmer la présence d'une cloison à l'arrière de la cavité et de retrouver les restes d'un adhésif qui a pu être analysé lui aussi (fig. 8). Il a été démontré que, si les yeux de verre des deux bustes relèvent de la même technique de fabrication, ils apparaissent toutefois distincts.

\section{La fusion de poudres de verre}

L'observation des yeux droits en verre de Livie et d'Auguste, à l'aide d'une loupe binoculaire, montre qu'ils ont été obtenus par le dépôt de poudres de verre dans un moule : du verre blanc opaque, correspondant à la cornée, est déposé sur le verre d'aspect noir placé au fond du moule, représentant l'iris. L'ensemble est alors introduit dans un four pour la fusion des poudres. La pupille est gravée dans le verre d'aspect noir. Cette gravure peut avoir été faite pendant la fabrication (dans le moule) ou bien par la suite, à froid, une fois les yeux réalisés. La surface du verre est dans les deux cas assez grumeleuse. Cette observation pourrait bien révéler l'état de surface des moules employés ou bien témoigner d'un début d'altération chimique en milieu d'enfouissement pour l'œeil de Livie, dont la teneur en sodium du verre d'aspect noir est relativement faible.

L'usage de poudres de verre est attesté par la grande quantité de bulles de gaz à l'intérieur du verre blanc, ainsi que par les contours irréguliers des interfaces entre les verres colorés, ce qui est particulièrement net pour l'œil de Livie. En effet, le cordon de verre gris qui encercle l'iris présente des frontières avec le verre blanc tout à fait caractéristiques d'une juxtaposition de grains de poudre de verre tout juste fondus (fig. 9) - que l'on peut observer par exemple dans 


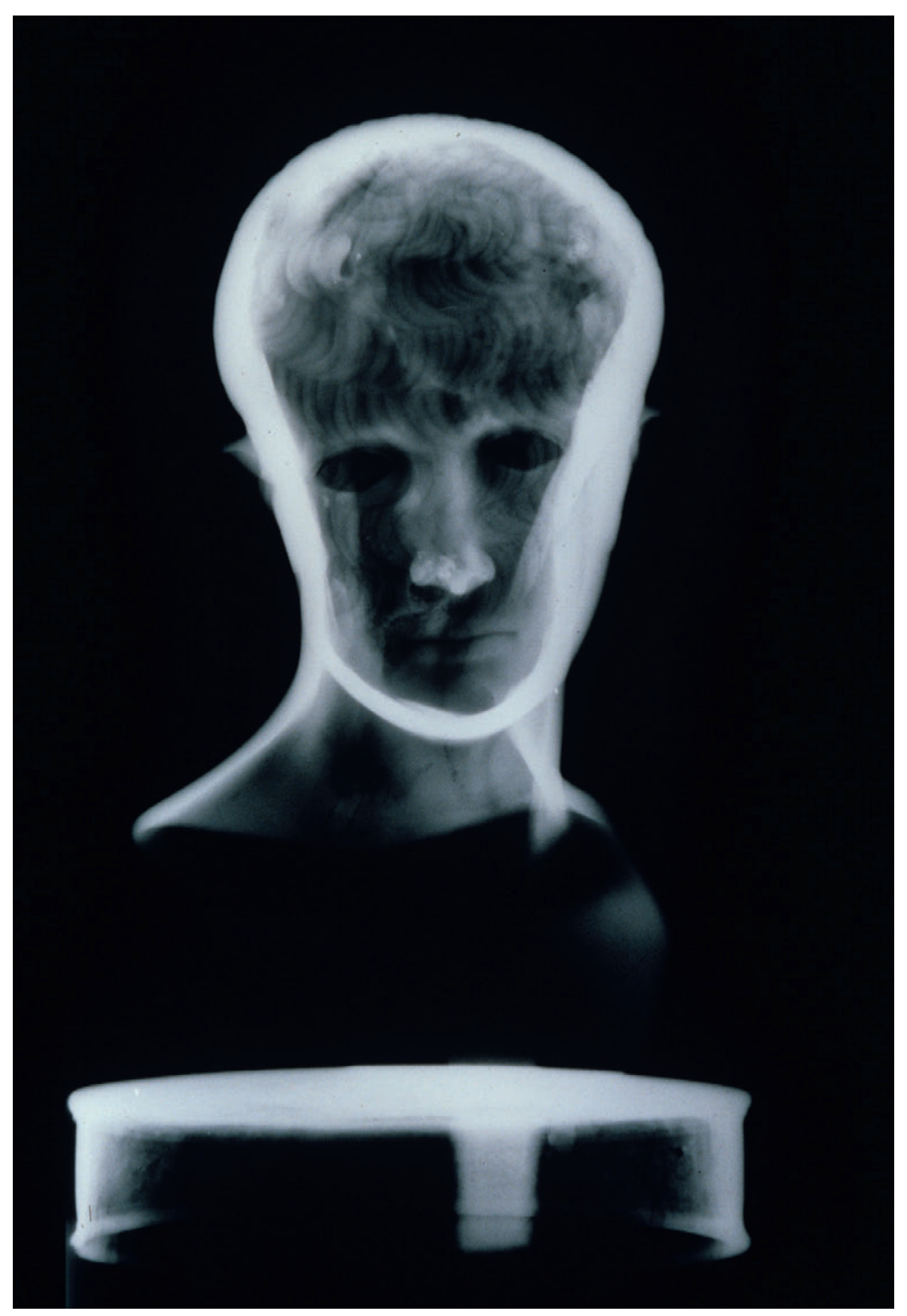

Fig. 4. Radiographie de face du buste d'Auguste, musée du Louvre, Br 29. (C) C2RMF/Th. Borel.

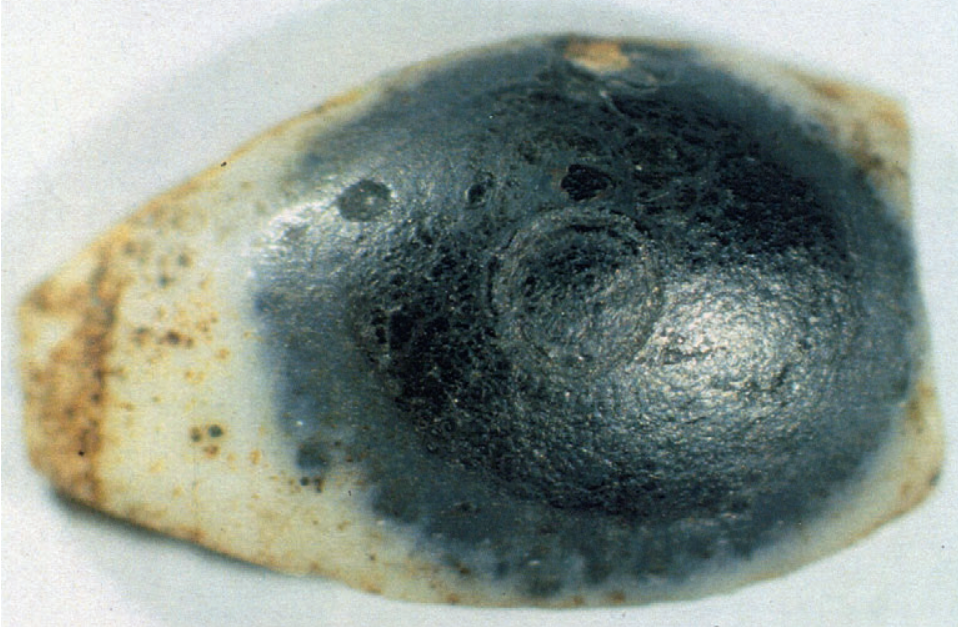

Fig. 6. CEil droit de Livie, verre blanc opaque et d'aspect noir, musée du Louvre, Br 28 (largeur de l'œil environ $1 \mathrm{~cm}$ ). (C) C2RMF/I. Biron.

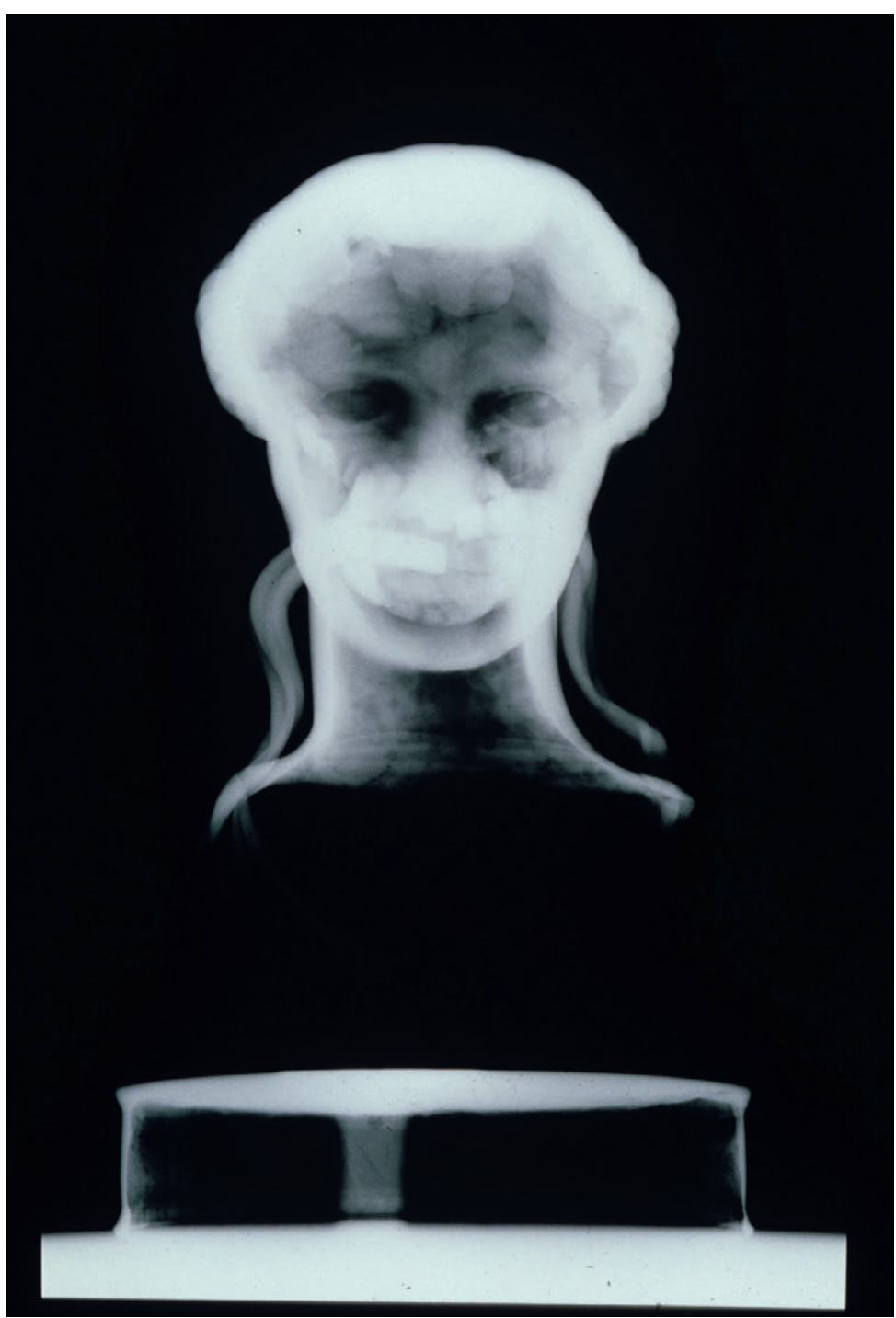

Fig. 5. Radiographie de face du buste de Livie, musée du Louvre, Br 28. (C) C2RMF/Th. Borel.

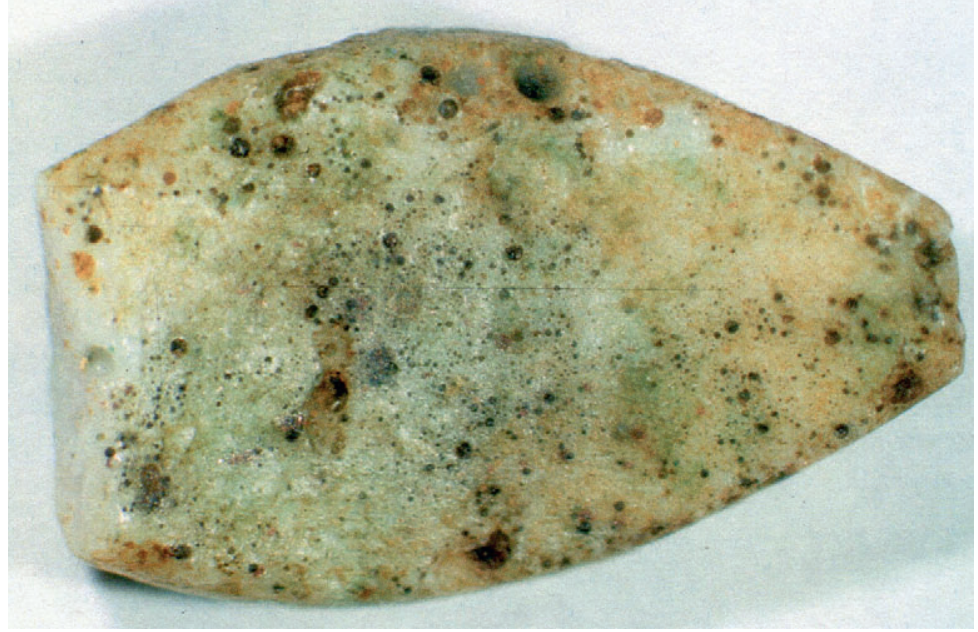

Fig. 7. Revers de l'œil droit de Livie, musée du Louvre, $\mathrm{Br} 28$ (largeur de l'œil environ $1 \mathrm{~cm}$ ). (C) C2RMF/I. Biron. 


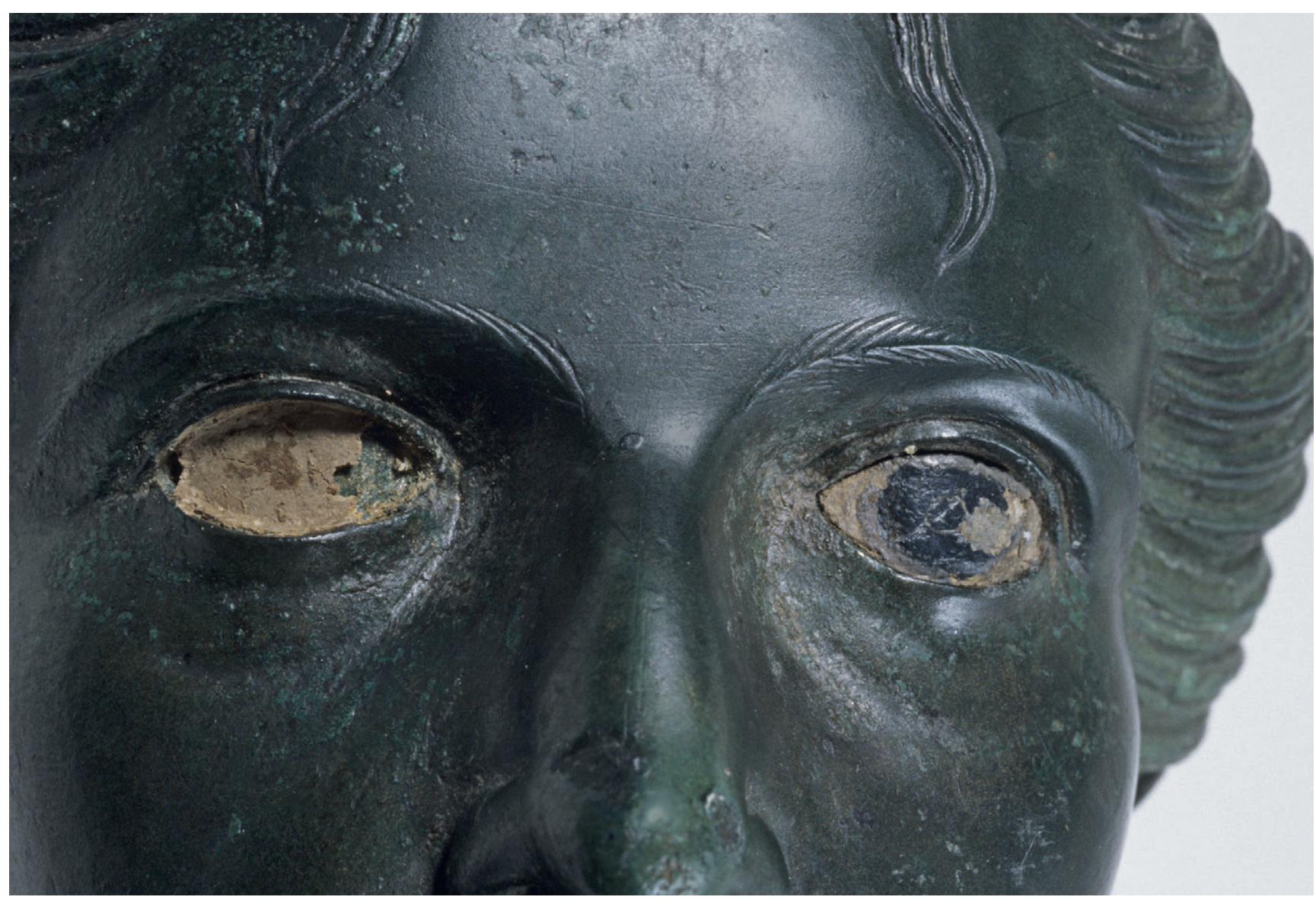

Fig. 8. Détail des yeux de Livie : l'œil droit en verre a été déchaussé pour étude au C2RMF ; la cavité laisse apparaître le ciment de fixation de couleur sable. L'oil gauche est restauré, musée du Louvre, Br 28. @ C2RMF/D. Bagault.

Tableau 1. Compositions chimiques élémentaires des verres constituant les yeux droits de Livie et d'Auguste obtenues par faisceau d'ions et exprimées en pourcentage massique d'oxydes. Valeurs moyennes de plusieurs points d'analyse. (C) C2RMF/I. Biron.

\begin{tabular}{|c|c|c|c|c|c|c|c|c|c|}
\hline$\%$ massique & $\mathrm{Na}_{2} \mathrm{O}^{*}$ & MgO & $\mathrm{Al}_{2} \mathrm{O}_{3}$ & $\mathrm{SiO}_{2}$ & $\mathbf{P}_{2} \mathbf{O}_{5}$ & $\mathrm{SO}_{3}$ & Cl & $\mathrm{K}_{2} \mathrm{O}$ & $\mathrm{CaO}$ \\
\hline \multicolumn{10}{|l|}{ Verres blancs opaques } \\
\hline LIVIE Br28 œil droit tranche & 13,60 & 0,58 & 3,23 & 62,02 & & & 0,69 & 0,84 & 7,07 \\
\hline AUGUSTE Br29 œil droit face & 16,49 & 0,72 & 2,50 & 56,28 & & & 0,66 & 0,96 & 7,24 \\
\hline \multicolumn{10}{|l|}{ Verre gris } \\
\hline LIVIE Br28 œil droit face & 14,45 & 0,63 & 3,18 & 64,90 & 0,10 & 1,00 & 1,01 & 1,91 & 7,97 \\
\hline \multicolumn{10}{|l|}{ Verres d'aspect noir } \\
\hline LIVIE Br28 œil droit face & 10,83 & 0,58 & 2,87 & 72,25 & 0,27 & 1,87 & 1,01 & 1,65 & 8,05 \\
\hline AUGUSTE Br29 oil droit face & 16,78 & 0,94 & 2,68 & 54,19 & 0,47 & 1,29 & 0,70 & 1,28 & 8,77 \\
\hline
\end{tabular}

* analyse par PIGE et autres éléments par PIXE. 
Fig. 9. CEil droit de Livie : détail de l'interface entre le verre blanc opaque et le verre d'aspect noir. Musée du Louvre, Br 28. (C) C2RMF/I. Biron.

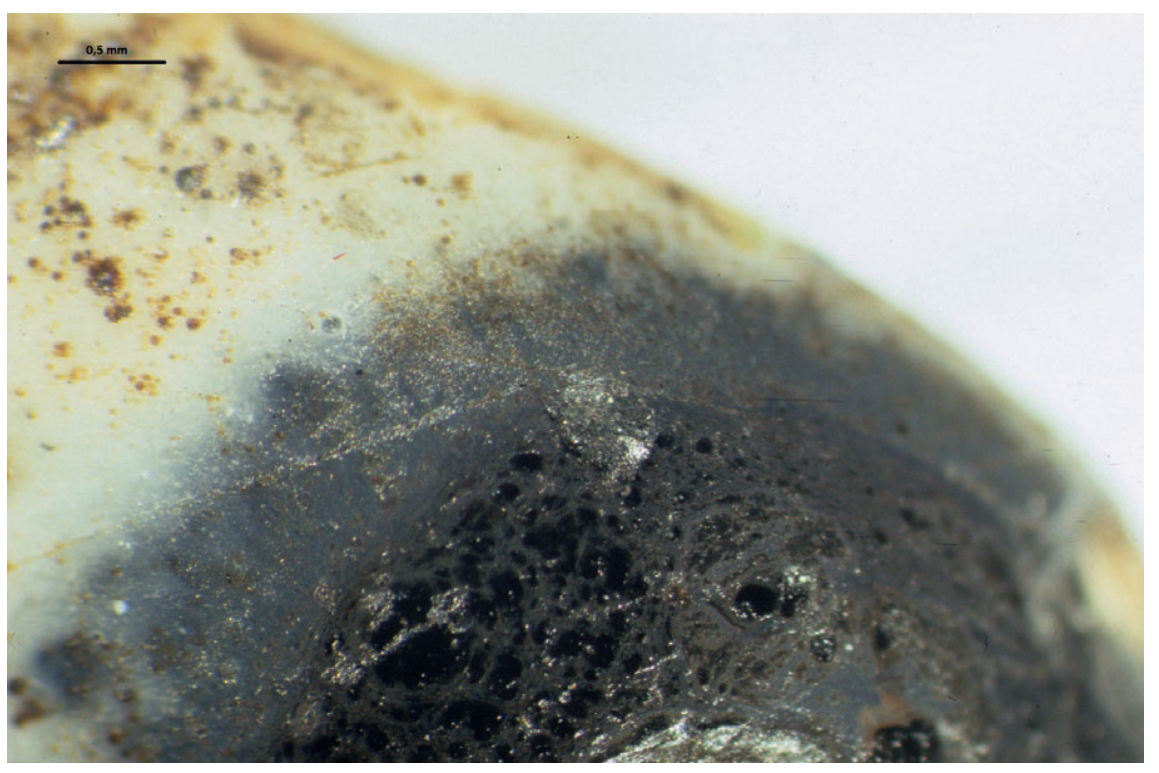

des émaux opaques du Moyen Âge ${ }^{23}$. D'après la forme des contours de ce liseré gris, la taille des grains de poudre employés pour l'œil de Livie peut même être estimée autour de $0,5 \mathrm{~mm}$ maximum.

\section{Une facture différente entre les deux bustes}

Les yeux se distinguent par deux points :

- les moules employés ne sont pas identiques, car la surface de l'œil d'Auguste est plus bombée que celle de Livie. L'œil droit de Livie est peu épais (environ 3-4 mm).

- deux verres colorés sont employés pour l'œil d'Auguste où le verre sombre jouxte directement le verre blanc opaque, tandis que trois verres colorés sont observés pour l'œil de Livie avec un cordon de verre gris présent à l'interface du verre d'aspect noir et du verre blanc opaque. Cette interface est volontaire. Il est donc probable que l'artiste, dans un souci de réalisme, ait voulu représenter le vieillissement naturel des yeux en accord avec l'âge de Livie. En effet, ce liseré de verre gris est trop large (entre 1 et $2 \mathrm{~mm}$ ) et trop homogène pour correspondre à une inter-diffusion des verres blancs et d'aspect noir pendant la cuisson. Il s'agit bien d'un mélange délibéré de poudres de verre sombre et blanc déposé autour de l'iris. Ce mélange est confirmé par les analyses chimiques (voir tableau 1).

La nature des verres employés pour les yeux de Livie et d'Auguste

La surface des verres employés pour fabriquer les yeux des bustes de Livie et d'Auguste a été analysée par faisceau d'ions en mode PIXE et $\mathrm{PIGE}^{24}$ de façon non-destructive avec l'accélérateur de particules AGLAE du C2RMF ${ }^{25}$. Différents points d'analyse sont réalisés pour chaque verre coloré et les valeurs moyennes sont reportées, en pour cent massique d'oxydes, dans le tableau ci-joint (tableau 1). Les conditions expérimentales sont données en note ${ }^{26}$.

\begin{tabular}{|c|c|c|c|c|c|c|c|c|c|c|}
\hline $\mathrm{TiO}_{2}$ & MnO & $\mathrm{Fe}_{2} \mathrm{O}_{3}$ & $\mathrm{CoO}$ & $\mathrm{NiO}$ & $\mathrm{CuO}$ & $\mathrm{ZnO}$ & $\mathrm{SrO}$ & $\mathrm{SnO}_{2}$ & $\mathrm{Sb}_{2} \mathrm{O}_{3}$ & $\mathrm{PbO}$ \\
\hline 0,11 & 1,49 & 0,57 & & 0,005 & 0,038 & 0,007 & 0,025 & 0,023 & 1,736 & 7,325 \\
\hline 0,16 & 1,36 & 0,57 & & 0,005 & 0,069 & 0,008 & 0,038 & 0,063 & 2,793 & 9,701 \\
\hline 0,14 & 0,79 & 0,63 & & 0,003 & 0,060 & 0,006 & 0,024 & 0,014 & 0,507 & 2,561 \\
\hline 0,07 & 0,03 & 0,41 & & 0,001 & 0,013 & 0,003 & 0,020 & & 0,005 & 0,057 \\
\hline 0,16 & 10,59 & 0,84 & 0,012 & 0,009 & 0,482 & 0,017 & 0,068 & 0,086 & 0,087 & 0,539 \\
\hline
\end{tabular}




\section{Les verres blancs opaques}

Les analyses montrent que la composition de ces verres, entre les deux yeux droits, est très proche, mais non identique. Ce sont des silicates sodo-calciques dont les sources sodiques sont d'origine minérale (natron), d'après les faibles teneurs en magnésium, en potassium et en phosphore du verre de base $^{27}$. Ils sont colorés et opacifiés par la présence de petits cristaux d'antimoniates de calcium répartis dans la matrice vitreuse $^{28}$. Si ces compositions présentent toutes les caractéristiques des verres romains, elles apparaissent cependant marginales au sein de cette production par leurs fortes teneurs en plomb de 7,3 et 9, 7\%. De telles concentrations ne sont observées que pour quelques types d'objets utilisant du verre blanc opaque. C'est le cas de la plupart des verres blancs opaques, décorant, selon la technique du camée (Roman cameo vessel glass), les verres de vaisselle bleus ou pourpres transparents produits entre le I ${ }^{\text {er }}$ siècle avant et le $\mathrm{I}^{\text {er }}$ siècle après J.-C. (la majorité entre 27 av. et 68 ap. J.-C.), comme le vase Portland du British Museum daté de la fin du $\mathrm{I}^{\mathrm{er}}$ siècle avant J.-C. ${ }^{29}$ Ces compositions se retrouvent aussi dans quelques verres blancs millefiori ou mosaïqués (mosaic glass) datant de la période hellénistique (fin du III $^{\mathrm{e}}$ à la fin $\mathrm{du} \mathrm{II}^{\mathrm{e}}$ siècle av. J.-C.) et de la période impériale (à partir du milieu du I ${ }^{\mathrm{er}}$ siècle av. J.-C. $)^{30}$.

Les teneurs en plomb dans ces verres varient de moins de $10 \%$ à parfois plus de $20 \%$, mais se situent le plus souvent entre 10 et $15 \%^{31}$. Si l'ajout de plomb dans un verre de base romain peut s'expliquer par la technique des camées, en rendant le verre plus facile à tailler ou en abaissant sa température de fusion ${ }^{32}$, il est en revanche plus difficile à comprendre pour les verres millefiori, comme pour les yeux de Livie et d'Auguste, à moins d'envisager leur fabrication dans des ateliers communs.

Cette production de verres colorés opaques décline au cours du $\mathrm{I}^{\mathrm{er}}$ siècle pour les verres de vaisselle au profit des verres soufflés incolores ou colorés naturellement, mais se poursuit cependant dans les mosaïques ${ }^{33}$. Les verres des yeux de Livie et d'Auguste proviennent vraisemblablement d'ateliers de vaisselle et non pas d'ateliers de mosaïstes. En témoigne la similitude des compositions entre les verres blancs utilisés pour réaliser les yeux de Livie et d'Auguste et les verres de vaisselle colorés décorés de camées et de millefiori, alors que les verres blancs opaques des tesselles de mosaïque sont exempts de plomb. Ces verres blancs colorés et opacifiés à l'antimoine contenant de fortes teneurs en plomb n'apparaissent qu'au début de la période romaine - pour la majorité d'entre eux du I ${ }^{\text {er }}$ siècle avant J.-C. au I ${ }^{\text {er }}$ siècle après J.-C., - et ne semblent pas communs ensuite ${ }^{34}$.

\section{Les verres d'aspect noir}

D’après les analyses, ces verres sont des silicates sodo-calciques dont les sources alcalines et les agents colorants diffèrent entre les deux bustes. L'apport de sodium provient de source minérale (natron) pour l'œil de Livie et probablement de source végétale (cendres de plantes) pour celui d'Auguste. Ce dernier contient en effet des teneurs relativement fortes en phosphore et en calcium, bien que celles en magnésium et potassium soient assez faibles. Par ailleurs, le verre gris de l'œil de Livie est également un silicate sodo-calcique dont les sources sodiques sont vraisemblablement issues de natron avec, sans doute, un faible apport de potassium (provenant de cendres?).

Toujours d'après ces analyses, le verre d'aspect noir de Livie est coloré avec du fer $(0,41 \%)$, probablement à l'aide du complexe fer-soufre qui produit une couleur allant de l'ambre au marron foncé selon les conditions d'oxydoréduction dans le bain lors de la fabrication ${ }^{35}$, alors que celui d'Auguste l'est avec du manganèse en forte teneur (10,59 \%) dont la couleur est pourpre foncé. Le fort pouvoir colorant de ces éléments, auquel s'ajoute l'épaisseur du verre (en particulier pour Livie) donnent l'impression que ces verres noirs sont opaques, mais ils sont en réalité transparents. Le verre gris de Livie est un mélange de verre blanc opaque et de verre sombre colorés avec probablement du fer $(0,63 \%)$ et du manganèse $(0,79 \%)$.

Les verres d'aspect noir romains ont fait l'objet de recherches archéologiques ${ }^{36}$ et d'analyses chimiques ${ }^{37}$ depuis seulement une quinzaine d'années. Cette production assez marginale au sein des verres romains fut néanmoins largement diffusée au cœur de l'Empire et concerne essentiellement des verres de vaisselle et des bijoux. Les yeux de Livie et d'Auguste semblent particulièrement connectés à la production de vaisselle qui apparaît dans tout l'Empire vers 30 à 70-80 après J.-C. ${ }^{38}$ Ces verres de vaisselle réapparaissent ensuite au cours de deux périodes plus tardives, régionalement dans les provinces du nord-ouest (Gallia Belgica, Germania Inferior et superior) de $170-180$ à 230-250 ${ }^{39}$ et enfin de nouveau dans le sud-est de la Méditerranée au cours des IV ${ }^{\mathrm{e}}$ et V $\mathrm{V}^{\mathrm{e}}$ siècles ${ }^{40}$. Les bijoux sont quant à eux produits pendant toute l'époque impériale, mais deviennent très courants dans tout l'Empire à partir de la seconde moitié du $\mathrm{II}^{\mathrm{e}}$ siècle ${ }^{41}$.

Quatre recettes principales sont recensées dans ces verres romains. Les deux qui furent employées jusqu'au milieu du $\mathrm{II}^{\mathrm{e}}$ siècle sont proches de nos analyses des yeux, mais ne leur correspondent pas vraiment, tant par la nature des sources alcalines que par la concentration en élément colorant. Il s'agit : de verres au natron colorés au manganèse (2-4\%) de couleur pourpre foncé (recette 1) et de verres aux cendres de plantes ou avec un mélange de cendres et de natron colorés naturellement par le fer apporté par les sables (1-2\%) de couleur vert foncé (recette 2). À partir du milieu du II siècle, une nouvelle recette de verres au natron colorés avec de fortes teneurs en fer apparaît (5-10\%, voire plus) (recette 3), puis à la fin du III $^{\mathrm{e}}$ siècle une autre très proche, mais employant des sables différents de couleur vert foncé (recette 4). Les verres au manganèse sont minoritaires par rapport aux autres au cours de cette période.

Bien que différent de ces recettes dominantes, le verre d'aspect noir de Livie correspond pourtant bien à une composition de verre romain. Dans l'important corpus de 


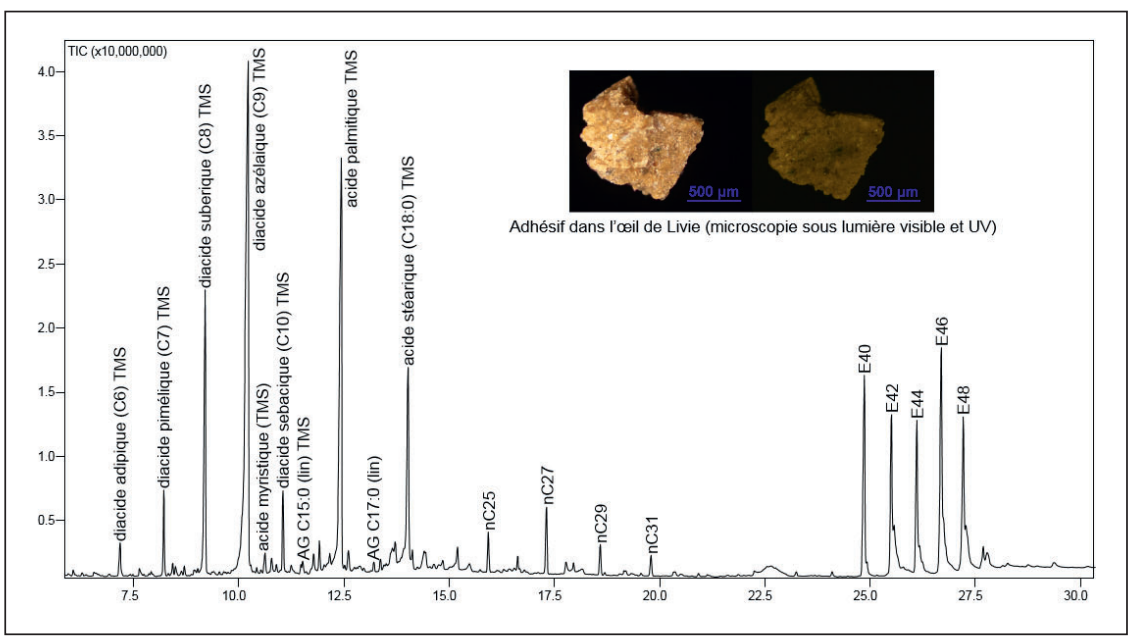

Fig. 10. Analyse chromatographique de l'adhésif sous l'œil de Livie. (c) C2RMF/J. Langlois.

verres romains d'aspect noir qui a été jusqu'à présent analysé (autour de 400 échantillons), quelques-uns se démarquent en effet de cet ensemble avec l'usage de recettes différentes. Jusqu'au milieu du $\mathrm{II}^{\mathrm{e}}$ siècle, certains verres sont en effet colorés avec du fer en faibles teneurs probablement par le complexe fer-soufre (couleur marron foncé) et d'autres avec des teneurs en cobalt et/ou en cuivre (couleur bleu-vert) ${ }^{42}$. La composition du verre d'aspect noir de Livie s'accorde bien avec certaines d'entre elles, qui sont des verres au natron contenant de faibles teneurs en fer comprises de 0,4 à $0,9 \%{ }^{43}$. En revanche, le verre d'Auguste se distingue de cet ensemble par sa très forte concentration en manganèse et par la présence de faibles teneurs en cuivre $(0,48 \%)$ et en cobalt (115 ppm), ainsi qu'en plomb $(0,54 \%)$, en antimoine (865 ppm) et en en étain (856 ppm). Ces derniers éléments impliqués dans les colorants (cuivre, cobalt), ou dans les opacifiants (plomb, antimoine et étain) révèlent l'emploi d'un mélange de verres de récupération.

Les analyses chimiques élémentaires des verres blancs opaques et d'aspect noir des yeux apportent un nouvel éclairage à notre connaissance des bustes. Elles permettent non seulement de situer la période de fabrication des yeux au cours du I ${ }^{\mathrm{er}}$ siècle de notre ère (et probablement entre 30 et $70^{44}$ ), mais aussi de confirmer leur appartenance à la production verrière romaine. Elles soulignent également le caractère indépendant de la facture des yeux entre les bustes et nous aident à envisager leur réalisation par des artisans produisant des verres de vaisselle. Enfin, le caractère marginal de ces compositions illustre de nouveau les particularités de la production verrière romaine, réputée fort homogène.

\section{Le mode de fixation des yeux}

L'œil droit de Livie ayant été déposé, la restauratrice en charge de l'intervention a pu prélever l'adhésif original de fixation dans la cavité ${ }^{55}$ (fig. 8). Des analyses en GCMS (Gaz Chromatography - Mass Spectrometry) ont été réalisées afin d'identifier les constituants moléculaires caractéristiques de cet adhésif. Ces analyses permettent de déceler une gamme assez large de composés naturels utilisés dans le patrimoine (résines, cires, matières grasses animales ou végétales...) et particulièrement bien adaptés à l'analyse des matériaux cireux et résineux ${ }^{46}$.

La nature des esters palmitiques identifiés ainsi que leur répartition sont caractéristiques de la présence d'une cire d'abeille. En plus de ces composés, la cire d'abeille contient à l'origine une série de n-alcanes à nombre impair d'atomes de carbone (entre 23 et 33), parmi lesquels l'heptacosane (noté nC27), qui est le composé majoritaire et une série d'acides gras linéaires (de 24 à 34 atomes de carbone). Seule la série d'alcanes reste visible ici, à l'état de traces par rapport à la composition originelle de la cire d'abeille. Les acides gras linéaires ont, quant à eux, complètement disparu (fig. 10).

Ces deux phénomènes de perte des éléments les plus légers de la cire d'abeille sont fréquents pour des matériaux antiques et dépendent du contexte de conservation des vestiges archéologiques. Il s'agit d'une altération partielle de la composition native de la cire d'abeille. Ces phénomènes d'altération peuvent témoigner d'un processus de dégradation ou de migration en surface, puis de sublimation de ces composés, selon le contexte de leur conservation d'origine. Une hydrolyse partielle des esters est également visible de par la présence de traces d'esters à 36 et 38 atomes de carbone ${ }^{47}$.

La série de diacides linéaires, allant de 6 à 10 atomes de carbone (l'acide azélaique en C9, étant majoritaire), associée à la présence des acides palmitique, stéarique et oléique, témoigne, quant à elle, de la présence d'huile végétale siccative.

Les résultats obtenus sont caractéristiques d'une cire d'abeille altérée mêlée à une huile végétale siccative. Ces deux substances naturelles sont des matériaux disponibles et fréquemment utilisés dans l'Antiquité. Leur présence ici est donc compatible avec la période de fabrication des bustes, même si elles ont également été largement utilisées par la suite et le sont encore de nos jours. 


\section{Bilan de l'étude}

\section{La mise en évidence d'une nouvelle technique antique d'élaboration des yeux}

On savait jusqu'à présent, par l'examen des bustes en bronze de petites dimensions de la fin de l'époque républicaine et des premières années de l'époque impériale, que les yeux pouvaient être rapportés en argent ${ }^{48}$. Ils pouvaient également être coulés directement avec la tête. Sur le portrait d'homme anonyme d'époque augustéenne, conservé au musée du Louvre (Br 21) et mentionné ci-dessus, c'est une cavité conique, ménagée au centre des yeux de bronze, une cavité peut-être regard $^{49}$. Les yeux pouvaient aussi être fabriqués, comme ceux de la grande statuaire de bronze, par un artisan spécialisé, un faber oculariarius, dont le statut était comparable à celui d'un orfèvre à cause de la préciosité des matériaux utilisés. Les orbites, désormais vides, de plusieurs portraits témoignent non seulement de la fragilité du dispositif, mais aussi de l'avidité des pilleurs antiques ${ }^{50}$. Les yeux préservés de quelques grands bronzes confirment le raffinement extrême de telles compositions. Le globe oculaire en marbre, en ivoire ou en albâtre était évidé en son centre pour accueillir l'iris et la pupille, cerclés de verre, de métal ou d'un anneau de pierre. Pour permettre à la lumière de traverser la matière, l'iris était en verre coloré. Les exemplaires les plus remarquables datent des $\mathrm{IV}^{\mathrm{e}}$ et $\mathrm{III}^{\mathrm{e}}$ siècles avant notre ère. Vers 300 avant J.-C., les iris mordorés des yeux de Seuthès III, incrustés dans de l'albâtre, ont été obtenus en mêlant du fer à la poudre de verre ; les pupilles, du fer et du manganèse. Les iris sont cerclés d'un anneau, qui a été coloré en mêlant du cuivre et un peu de fer à la poudre de verre ${ }^{51}$. Peu avant 25 avant J.-C., l'iris vert de l'Auguste de Méroé, incrusté dans le globe oculaire en marbre, est constitué de plusieurs éléments de verre en forme de coins, qui sont juxtaposés ${ }^{52}$. Et c'est bien là ce qui distingue ces yeux partiellement en verre de ceux d'Auguste et de Livie. Pour les deux bustes du Louvre, il ne s'agit pas d'éléments indépendants, qui composent à la manière d'une mosaïque les différentes parties de l'œil. Aucune césure matérielle ne marque le passage entre la sclérotique blanche et les iris sombres. Les iris ont été fondus avec le globe oculaire dans un moule qui donne à l'œil une forme plus ou moins convexe ; les pupilles sont notées par un petit cercle incisé. L'identification, pour les yeux de portraits en bronze, de techniques verrières romaines de luxe - celle de la vaisselle colorée décorée de camées ; celles des verres millefiori et des bijoux - apporte un témoignage nouveau sur les liens qui devaient nécessairement exister entre ateliers et artisans producteurs d'objets précieux.

Une technique certainement loin d'être isolée

Cette technique, à la fois virtuose et exceptionnelle, a été identifiée, à la suite de l'étude des yeux d'Auguste et de Livie, sur un troisième bronze conservé au musée J. Paul Getty de Los Angeles : l'observation sous microscope des yeux de verre d'un portrait de jeune fille a confirmé en effet qu'ils avaient bien été fabriqués selon le même procédé (fig. 11) ${ }^{53}$. Par ailleurs, la cavité peu profonde de l'œil de Livie comporte, nous l'avons dit, une cloison fermée. Sans doute faut-il interpréter cette paroi verticale comme un indice nouveau, susceptible d'aider à repérer d'autres bronzes qui auraient pu bénéficier d'une technique comparable pour la fabrication de leurs yeux. Pouvait-elle être utilisée pour des bronzes de grandes dimensions? C'est l'hypothèse formulée récemment à propos d'un Apollon et d'une Diane de Pompéi, qui présentent une telle fermeture au fond de leurs orbites ${ }^{54}$.

\section{Un critère de datation}

L'étude des yeux d'Auguste et de Livie permet d'apporter une réponse à la question longuement débattue de la datation des deux portraits, avant ou après la mort de l'empereur, et de lever les interrogations suscitées par la lecture de la titulature ambiguë du couple impérial. D’après les résultats des analyses du verre, les yeux d'Auguste et de Livie s'inscrivent dans une fourchette chronologique allant des environs de 30 à 70-80 ap. J.-C. L'insistance sur le caractère vieillissant des empereurs pourrait alors signifier que les bustes ont été produits au plus tôt dans les dernières années du règne de Tibère, fils de Livie et successeur d'Auguste. La confirmation de la nature des yeux de la jeune fille du Getty conforterait aussi l'hypothèse, formulée à partir de la coiffure, de sa datation à l'époque de Tibère.

Une question demeure ouverte, celle de la destination des deux portraits du Louvre. Ont-ils été déposés dans un laraire ou consacrés dans un temple ? La présence d'inscriptions votives, désormais authentifiées par la découverte récente d'une troisième dédicace mentionnant un Atespatus, irait plutôt dans le sens de la seconde hypothèse ${ }^{55}$. Quoi qu'il en soit, il s'agissait d'une démarche privée. Le dédicant des deux bustes était un notable indigène, qui n'avait pas la citoyenneté romaine, mais adhérait aux valeurs de Rome.

\section{Remerciements}

Nous tenons à exprimer notre très sincère reconnaissance à Brigitte Bourgeois, qui nous a donné la possibilité de publier cet article. Nos remerciements vont également aux membres de l'équipe AGLAE - Claire Pacheco, Quentin Lemasson, Laurent Pichon et Brice Moignard - pour leur assistance technique lors des analyses du verre, ainsi qu'aux restauratrices Agnès Conin et Juliette Dupin. 


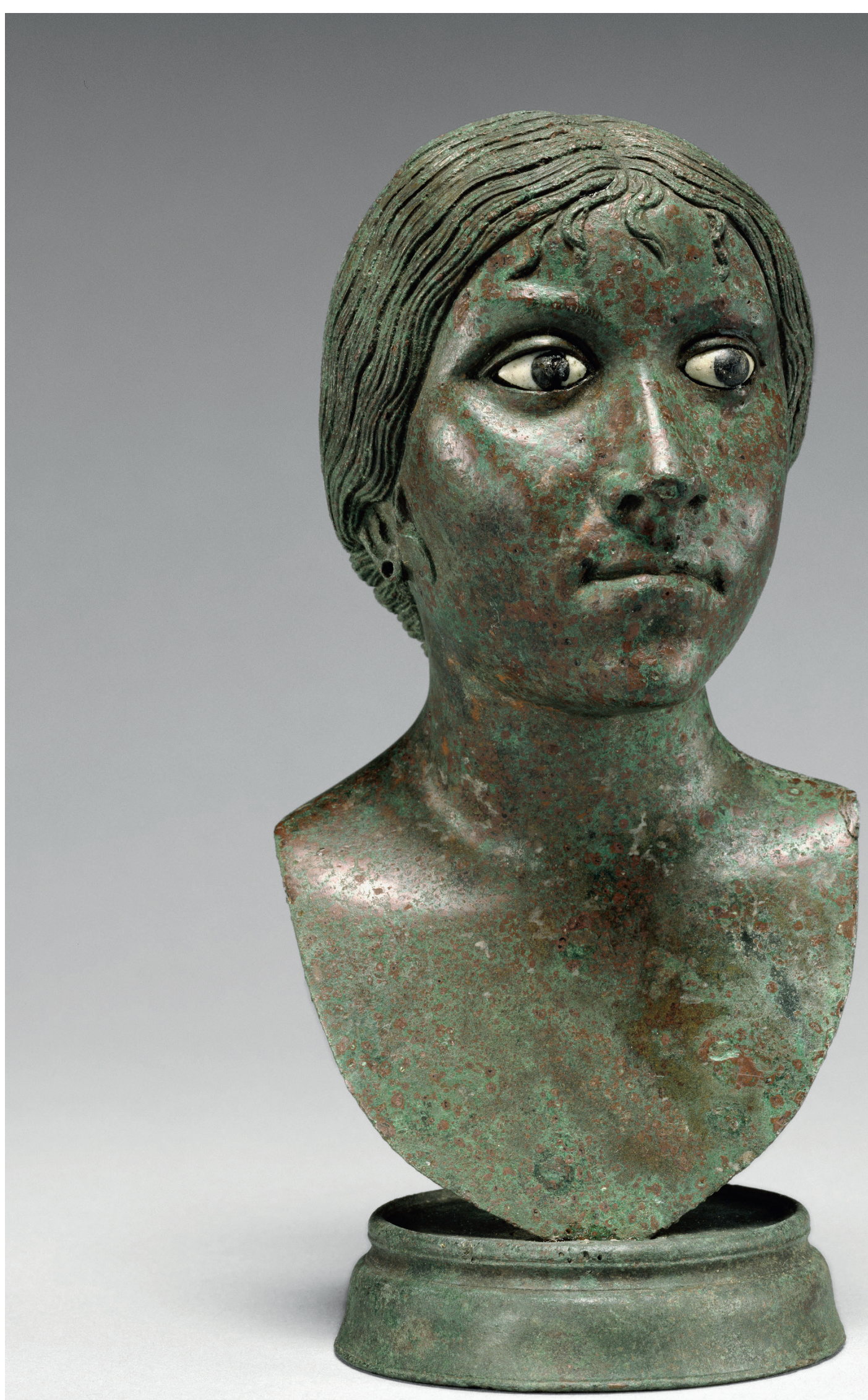




\section{Le dédicant Atespatus : un nom assuré}

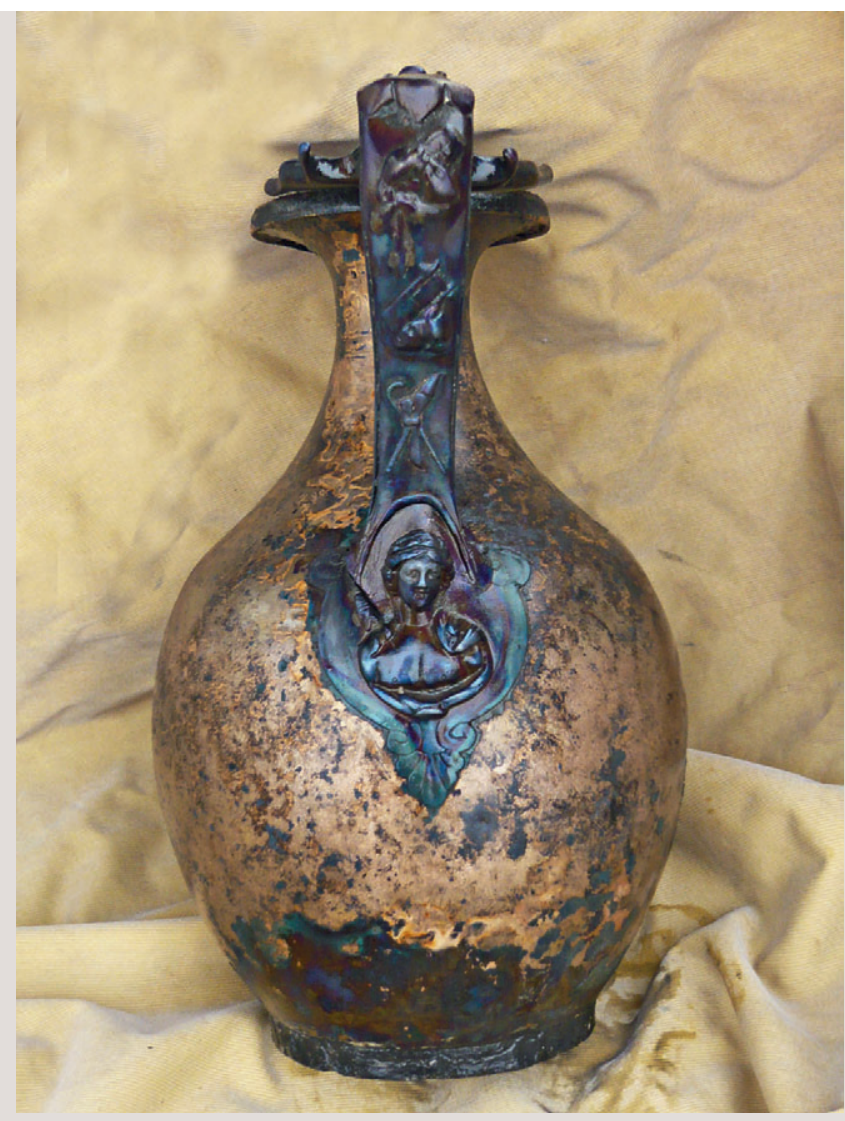

Fig. 1. Vase de Saint-Ambroix-sur-Arnon. @ C. Cribellier.

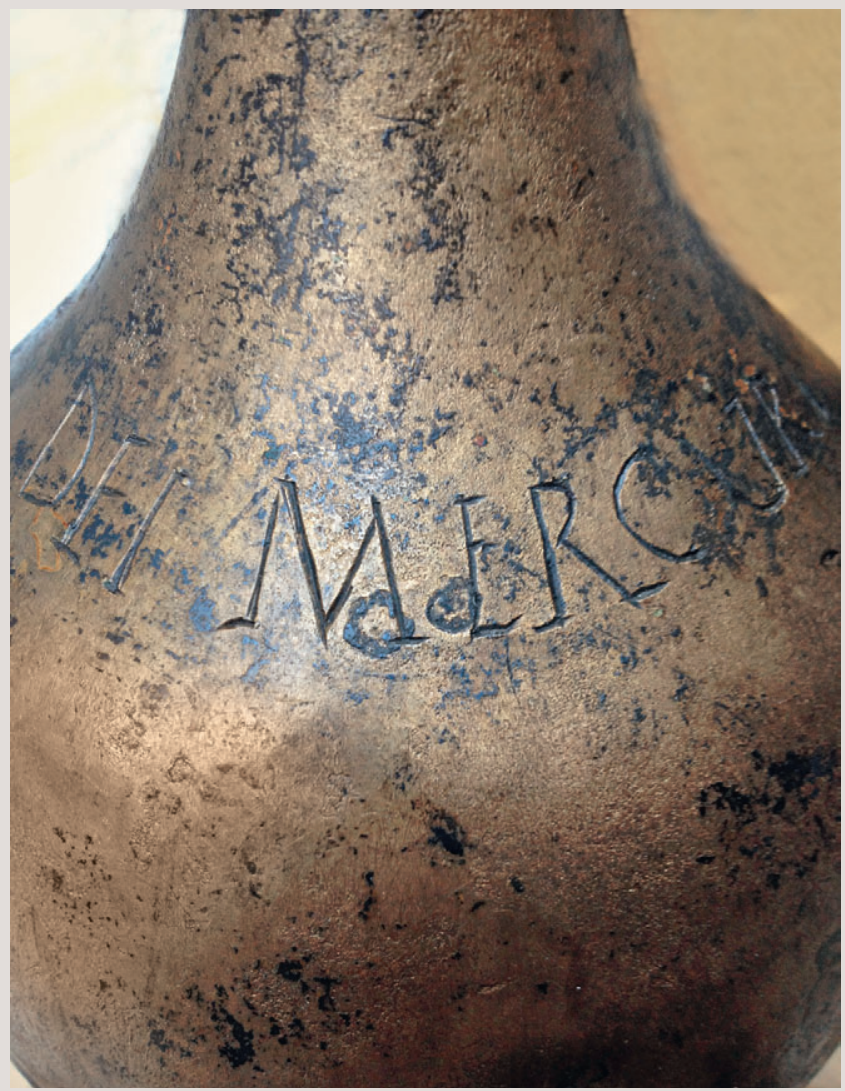

Fig. 2. Détail du nom « Mercuri ». () C. Cribellier. 


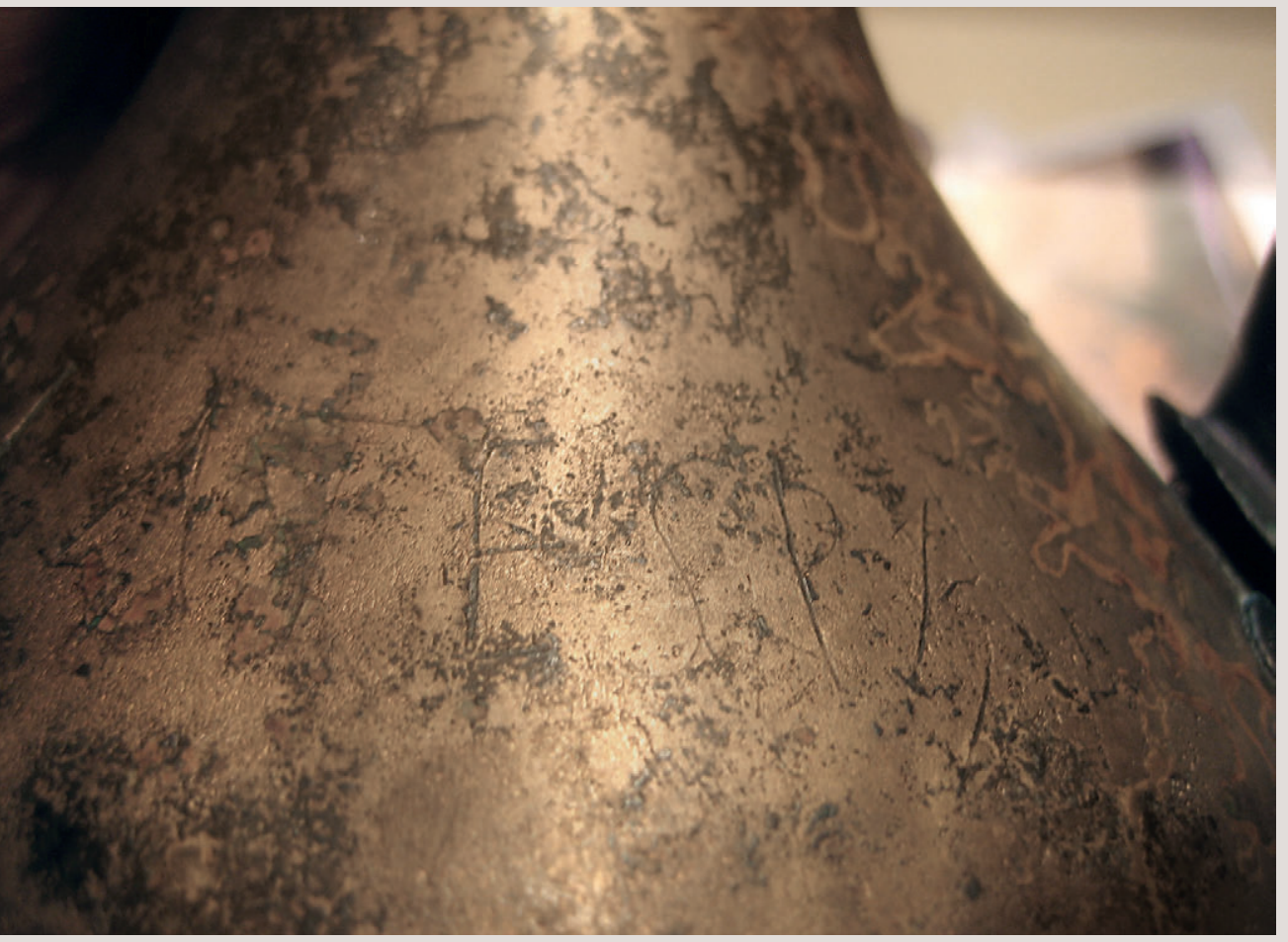

Fig. 3. Détail du nom «Atespatus ». (C) B. Bertin.

\section{Notes}

\section{CIL XIII, 1366}

2. Province d'Aquitaine, à la limite entre les départements du Cher et de l'Indre, région Centre. Cribellier, 2014, p. 52.

3. Le S final a disparu, parce qu'il était trop superficiellement marqué, ou parce que, trop près de l'anse, il a été effacé par sa soudure ou par la rouille. Voir DondinPayre, "Les vases du sanctuaire de La Vallée de Saint-Ambroix », ibid., p. 80.

\section{Bibliographie}

CIL XIII : Corpus Inscriptionum Latinarum. Inscriptiones trium Galliarum et Germaniarum Latinae, O. Hirschfeld (éd.), Berlin, 1899.

Cribellier C., 2014, « Le sanctuaire de La Vallée à Saint-Ambroix (Cher) » dans Exp. Argentomagus, p. 52.

Dondin-Payre M., 2014, « Les vases du sanctuaire de La Vallée de SaintAmbroix » dans Exp. Argentomagus, p. 80.

Exp. Argentomagus, 2014 : Dieux merci! Sanctuaires, dévots et offrandes en Gaule romaine [Exposition Saint-Marcel,

Musée d'Argentomagus, Argenton, 27 juin-7 décembre 2014, Fauduet I. (éd.)].

\section{Notes}

1. Sur le rôle de Prosper Mérimée, voir Héron de Villefosse, 1907, p. 8-9. Sur les avis opposés des spécialistes, voir par exemple Bartman, 1999, p. 3, 195-196 (modernes) ; Lahusen, Formigli, 2001, p. 70-71 (antiques).

2. Paris, musée du Louvre, département des Antiquités grecques, étrusques et romaines, Br 28 (Livie), Br 29 (Auguste) ; H. bustes $19 \mathrm{~cm}$ et $18,5 \mathrm{~cm}$; H. bases $3 \mathrm{~cm}$. Restauration 2005-2006 : A. Conin, J. Dupin, Frœhner, 1873 ; De Ridder, 1913, p. 11, $\mathrm{n}^{\text {os } 28-29,}$ pl. 5 ; Kersauson (de), 1986, $n^{\text {os }} 41-42$, p. 94-97 ; Bartman, 1999, p. 3, 195-196 ; Lahusen, Formigli, 2001, $\mathrm{n}^{\text {os }} 25-26$, p. 70-73, 360-363, 462, 466, 467, 472, 473 ; Dahmen, 2001, n ${ }^{\text {os }} 32-33$, p. 23-26, 53, 155, 156 ; Descamps, 2007 ; 2008 ; Szewczyk, 2014 .

3. Près de Moulin (Allier, France), CAG 03, 1989, n²60, p. 124. Le ruisseau correspond à La Sonnante : Letterlé, 2004.

4. Rolley, 1979, p. 16 ; AvisseauBroustet, 2002.

5. Acte notarié du 29 mai 1816. Bertrand, 1868-1869. Les bustes furent vendus à Pierre Saulnier, fils de la fermière des Guillemins, alors clerc de notaire à Moulins, puis cédés au propriétaire des lieux M. Girodet et restèrent dans sa famille plus de cinquante ans avant d'être vendus aux antiquaires parisiens Rollin et Feuardent (Frœhner 1973, p. 2), puis au Louvre en août 1868 avec l'accord de Napoléon III (n ${ }^{\text {os }}$ d'entrée NIII 3253 et NIII 3254).

6. Les yeux gauches ont été restaurés avant l'entrée des bustes au Louvre en septembre 1868 : Frohner, 1873, p. 5.

7. Respectivement $\mathrm{Br} 21$ et $\mathrm{Br} 42$.

8. Robbiola, Hurtel, 1995. L'historique des œuvres est inexact.

9. CIL XIII 1366. Sur le caractère honorifique des inscriptions, plutôt que témoignage d'une forme de culte impérial, voir Fishwick, 1991, p. 535 n. 363.

10. La hauteur depuis le menton jusqu'au sommet du crâne est de $10 \mathrm{~cm}$ pour Livie et de $11,5 \mathrm{~cm}$ pour Auguste.

11. Pour le type Louvre-Forbes, voir au musée du Louvre le portrait d'Auguste Ma 1280, possiblement daté de 29 av. J.-C. (Szewczyk, 2014, n 32, p. 78).

12. Le premier témoignage de cette mode capillaire se trouve au revers d'un aureus de Marc Antoine, daté des années 40-39 avant J.-C., sur lequel on identifie le profil d'Octavia. Berlin, Münzkabinett, Bartman, 1999, p. 59, fig. 47.

13. Buste éponyme du type dit de Marbury Hall, conservé au musée de Liverpool, inv. 1988.116, Bartman, 1999, p. 64-67, 161-162, fig. 52-54, 143.

14. Bartman, 1999, p. 67, 190, fig. 55.

15. D'après le buste éponyme découvert à Arsinoé et conservé à la Ny Carlsberg Glyptotek de Copenhague, inv. n ${ }^{\circ} 1444$, Johansen, 1994, n 36, p. 96-97 (pas de mèches parotides) ; Bartman, 1999, p. 4-5, 74- 77, 174-175.

16. Paris, musée du Louvre, Département des Antiquités grecques, étrusques et romaines, Ma 1233.

17. Ny Carlsberg Glyptotek, inv. $\mathrm{n}^{\circ} 748$, Johansen, 1994, n 35, p. 94-95 (mais 
présence d'une tresse qui entoure la tête). 18. Bartman, 1999, p. 90, fig. 75.

19. Lahusen, Formigli, 2001, p. 73, 472-473 (tableaux avec la composition élémentaire des bustes, d'après les analyses de L. Hurtel au C2RMF (C2RMF, dossier F218021, rapport $n^{\circ}$ Z1589, 1989), alors appelé Laboratoire de Recherche des Musées de France (LRMF), en \% massique) : Buste d'Auguste : $0,005 \mathrm{Zn} ; 6,0 \mathrm{~Pb}$; $5,04 \mathrm{Sn} ; 0,02 \mathrm{As} ; 0,326 \mathrm{Sb} ; 0,004 \mathrm{Fe} ; 0,146$ $\mathrm{Ag} ; 0,038 \mathrm{Ni} ; 0,053 \mathrm{Bi} ; 0,0001 \mathrm{Co}$. Socle d'Auguste : $0,01 \mathrm{Zn} ; 5,8 \mathrm{~Pb}$; 5,7 Sn ; 0,022 As ; 0,35 Sb ; 0,004 Fe ; 0,156 $\mathrm{Ag} ; 0,046 \mathrm{Ni} ; 0,005 \mathrm{Bi} ; 0,0006 \mathrm{Co}$. Buste de Livie : $0,011 \mathrm{Zn} ; 5,2 \mathrm{~Pb} ; 5,7$ Sn ; 0,014 As ; 0,332 Sb ; 0,004 Fe ; 0,086 Ag ; $0,043 \mathrm{Ni} ; 0,005 \mathrm{Bi} ; 0,0001 \mathrm{Co}$. Socle de Livie : $0,008 \mathrm{Zn} ; 5,0 \mathrm{~Pb} ; 5,7$ $\mathrm{Sn} ; 0,017 \mathrm{As} ; 0,31 \mathrm{Sb} ; 0,004 \mathrm{Fe} ; 0,16 \mathrm{Ag}$; $0,04 \mathrm{Ni} ; 0,005 \mathrm{Bi} ; 0,0008 \mathrm{Co}$

20. Voir ci-dessus n. 11.

21. Bartman, 1999, p. 13, 187-188,

fig. 11, 181. 22. Dossier C2RMF FZ18020, rapport $\mathrm{n}^{\circ}$ 10635, Biron, Langlois, Bouquillon, Bagault, 2007.

23. Biron et al., 1995, 1998, 2015. 24. Respectivement émissions de rayons $\mathrm{X}$ et gamma induites par particules. 25. Trois détecteurs ont été utilisés en simultané (deux détecteurs de rayons $\mathrm{X}$ ( $\mathrm{Si}(\mathrm{Li})$ - géométrie $45^{\circ}$ et un de rayons gamma (HpGe) - géométrie avant à $45^{\circ}$ ) pour accéder à la composition complète et précise du verre. Les résultats ont été dépouillés avec le logiciel Gupix (Campbell et al., 2010) et calibrés avec des échantillons de verre de référence (standards Corning Brill A, B, C et D et BGIRA 4 et 5 ).

26. Calligaro et al., 1996, Dran et al., 2004.

27. Composition du verre sans l'ajout éventuel de colorant, d'opacifiant et de plomb.

28. L'opacification est produite par la taille des cristaux et la différence d'indice de réfraction entre ces derniers et la matrice.

29. Bimson et Freestone, 1988.

30. Gedzeviciute et al., 2009.

31. Bimson et Freestone, 1988.

32. Le verre blanc était déposé en couche sur le support du vase, puis taillé mécaniquement ensuite.

33. Freestone et Stapleton, 2015.

34. Freestone et Stapleton, 2015.

35. Plus les conditions sont réductrices dans le bain en fusion et dans l'atmosphère du four, plus la couleur sera sombre, Freestone et Stapleton, 2015, Ceglia et al., 2014, Biron et Chopinet, 2013.

36. Cosyns et Hanut, 2005, Cosyns 2009, Cosyns et Fontaine, 2009.

37. Cosyns et al., 2012, Cagno et al., 2013a et b, Ceglia et al., 2014, Cagno et al., 2014.

38. Cosyns et Fontaine, 2009. 2006.

39. Cosyns et Hanut, 2005, Cosyns et al.,

40. Cosyns, 2011

41. Cosyns, 2011

42. Cagno et al., 2014.

43. Avec des teneurs en soufre

cependant plus faibles $(<0,5 \%)$.
44. Dénominateur commun entre les périodes de production importantes des verres blancs et ceux d'aspect noirs.

45. Un deuxième micro-prélèvement a été réalisé afin d'identifier l'adhésif de l'œil original (œil droit) du buste d'Auguste. Ce prélèvement entrepris dans le coin droit de l'œil, sur une fine bordure où l'adhésif est débordant de l'incrustation de verre, est constitué d'une cire d'entretien de stéarine appliquée sur le buste. Les signaux obtenus, très concentrés en éléments constitutifs de ce matériau cireux, témoignent également de son caractère plus moderne (la stéarine est synthétisée au cours du XIX ${ }^{\mathrm{e}}$ siècle) et éliminent toute chance d'identifier l'adhésif original.

46. Colinart, 1987 ; Regert et al., 2005.

47. Regert et al., 2001.

48. Portrait d'homme, Vienne, Kunsthistorisches Museum, inv. VI 273, Lahusen, Formigli, 2001, n ${ }^{\circ} 15$, p. 52, 53 , 350 , 467, fig. 15.2, 15.4, 15 d, 23 (yeux rapportés en argent) et p. 462 (synthèse des différentes possibilités).

49. Portrait d'homme, musée du Louvre, département des Antiquités grecques, étrusques et romaines, $\mathrm{Br} 21$, ci-dessus p. 87 et n. 7 ; Lahusen, Formigli, 2001, n 16 , p. 53, 54, 350, 351, fig. 16.1, 16.2 , $16.10,16 \mathrm{a}, 16 \mathrm{~b}, 16 \mathrm{~d}$

50. Portrait d'Auguste, Copenhague, Ny Carsberg Glyptotek, inv. 745, Lahusen, Formigli, 2001, n 19, p. 61, 356, fig. 19.1, 19.2, 19 a, 19 b ; portrait de femme âgée, Paris, musée du Louvre, département des Antiquités grecques, étrusques et romaines, Br 42, ci-dessus p. 87 et n. 7 ; Lahusen, Formigli, 2001, n ${ }^{\circ} 79$, p. 136-139, 378, fig. 79.1, 79.4, 79 a, 79 b.

51. Formigli, 2015, p. 10-12, fig. 20a-b, 22.

52. Lahusen, Formigli, 2001, p. 59, 355, 466 fig. 10-11, fig. 18. 14-15.

53. Malibu, The J. Paul Getty Museum, inv. 84. AB. 59, H. 16,5 cm ; Lahusen, Formigli, 2001, p. 95-96, fig. 44.1-4 ; Dahmen, 2001, p. 73 (époque de Tibère) ; site officiel du J. Paul Getty Museum avec bibliographie exhaustive : 25 B.C.-A.D. 25. Nous remercions très sincèrement David Saunders, Conservateur à la Villa Getty, d'avoir favorisé, à la demande de $\mathrm{S}$. Descamps, l'étude sous microscope des yeux du petit buste. Que Jerry Podany, responsable du service de restauration à la Villa Getty, qui a conduit cet examen en présence de S. Descamps à l'automne 2013, trouve ici l'expression de notre reconnaissance. Il a confirmé l'utilisation du même procédé. Notons l'absence de cils pour ce portrait comme pour ceux de Neuilly-le-Réal.

54. Les deux statues présentent bien des cavités oculaires peu profondes, fermées à l'arrière par des cloisons fondues avec les têtes. Toutefois, le blanc des yeux conservés de la Diane est en os. Risser, Saunders, 2015, p. 90-91, fig. 13a-c.

55. Kaufmann-Heinimann, 2004, p. $249-250$

\section{Bibliographie}

Avisseau-Broustet M., 2002, «Jeune Nubien ", dans Exp. Paris, 2002, n 57c, p. 143.

Bartman E., 1999, Portraits of Livia : imaging the imperial woman in Augustan Rome, Cambridge University Press, Cambridge.

Bertrand A., 1868-1869, "Procès-verbal des deux bustes en bronze d'Auguste et de Livie, à Neuilly-le-Réal (Allier) », Bulletin de la Société d'Émulation du Département de l'Allier, t. XI, p. 255-258.

Bimson M., Freestone I. C., 1988, "An analytical study of the relationship between the Portland vase and other Roman cameo", Journal of Glass Studies, 30, p. 55-64.

CAG 03, 1989, Corrocher J., Piboule M., Hilaire M., Carte archéologique de la Gaule 03. L'Allier, Paris.

Biron I., Chopinet M. H., 2013, "Colouring, decolouring and opacifying of Glass", in Janssens K. (dir.), Modern methods for analysing Archaeological and Historical glass, John Wiley \& Sons Ltd Publication, Chichester, West Sussex, Chap. 1. 3, I, p. 49-64.

Biron I., Dandridge P., Wypyski M. Th., 1995, «Le cuivre et l'émail ; technique et matériaux », dans Taburet-Delahaye E. et Boehm B. (dir.), L'œuvre de Limoges, émaux limousins du Moyen Âge, RMN, Paris, p. 48-62 et p. 446-449. Version anglaise : mêmes auteurs, 1996, "Techniques and Materials in Limoges Enamels", in Enamels of Limoges 11001350, The Metropolitan Museum of Art, New York, 48-62 et 445-449.

Biron I., Morel D., Borel Th., 1998, «Les triptyques reliquaires Dutuit : de l'œil du connaisseur à l'examen en laboratoire - histoire d'une réhabilitation ", Technè, 8, p. 97-106.

Biron I., avec la collaboration de TaburetDelahaye E., Durand J., Notin V. Crépin-Leblond Th., Bimbenet-Privat M., Dion-Tenenbaum A., Beillard B., 2015, Émaux sur métal du IX $X^{e}$ au $X I X^{e}$ siècle, histoire, technique et matériaux, éditions Faton, Dijon.

Cagno S., Cosyns P., Nys K., Janssens K., 2013a, "Black appearing Roman Glass", in Janssens K. (dir.), Modern methods for analysing Archaeological and Historical glass, John Wiley \& Sons Ltd Publication, Chichester, West Sussex, Chap. 5. 4, II, p. 368-385.

Cagno S., Cosyns P., Van der Linden V., Schalm O., Izmer A., Deconinck I., Vanhaecke F., Nowak A., Wagner B., Bulska E., Nys K., Janssens K., 2013b, "Composition data of a large collection of black appearing glass", Open Journal of Archaeometry, 1 : e22, p. 104-108, mis en ligne juin 2013, DOI: 10.4081/ arc.2013.e22, URL: https://www. researchgate.net/ publication/273338045.

Cagno S., Cosyns P., Izmer A., Vanhaecke F., Nys K., Janssens K., 2014, "Deeply colored and black-appearing Roman glass: a continued research", Journal 
of Archaeological Science, Technology E Provenance, 42, p. 128-139.

Calligaro Th., McArthur J. D., Salomon J., 1996, "An improved experimental setup for the simultaneous PIXE analysis of heavy and light elements with a $3 \mathrm{Mev}$ proton external beam", Nucl. Instr. Meth. Phys. Res B109/110, p. 125-128.

Campbell J. L., Boyd N. I., Grassi N., Bonnick P., Maxwell J. A., 2010, "The Guelph PIXE software package IV", Nuclear Instrument and Methods B, 268, 3356-3363.

Ceglia A., Nuyts G., Cagno S., Meulebroeck W., Baert K., Cosyns P., Nys K., Thienpont H., Janssens K., Terryn H., 2014, "A XANES study of chromophores: the case of black glass", Analytical Methods, The Royal Society of Chemistry, mis en ligne en mars 2014, DOI : 10.1039/c3ay42029a, URL : https://www.researchgate.net/ publication/260332475

CIL XIII : Corpus Inscriptionum Latinarum. Inscriptiones trium Galliarum et Germaniarum Latinae, pars II, fasc. 2, Inscriptiones Germaniae inferioris. Miliaria Galliarum et Germaniarum (Momsen Th., Hirschfeld O., Domaszewski A.), Berlin, 1907.

Colinart S., 1987, « Matériaux constitutifs », dans Gaborit J.-R. et Ligot J. (éds.), Sculptures en cire: de l'ancienne Égypte à l'art abstrait, RMN, Paris, p. 29-59.

Cosyns P., Hanut F., 2005, "Black Glass of second to third-century date in northern Gaul: a preliminary survey", in Annales du $16^{e}$ Congrès de l'AIHV-Association Internationale pour l'Histoire du Verre (London, 2003), Nottingham, p. 113118.

Cosyns P., Janssens K., Schalm O., Van der Linden V., 2006, "Black glass vessels and jewellery in the Roman Empire: a work in progress", in Creemers G., Demarsin B., Cosyns P. (dir.), Roman Glass in Germania Inferior. Interregional Comparisons and Recent Results, International colloquium Tongeren, 13/05/2005 (Atuatuca 1), Hasselt, p. $30-41$.

Cosyns P., 2009, "Sainte-Menehould (FR) and Trier (DE): two Roman workshops of black glass jewellery in the Northwest provinces reconsidered", in Annales du 17 congrès de l'AIHV (Antwerp, 2006), Antwerp University Press, p. 88-95.

Cosyns P., Fontaine S. D., 2009, « La vaisselle en verre d'apparence noire dans les provinces occidentales au I ${ }^{\mathrm{er}}$ siècle ap. J.-C. ", dans Annales du $17^{e}$ congrès de l'AIHV (Antwerp, 2006), Antwerp University Press, p. 80-87.

Cosyns P., 2011, "The production, distribution and consumption of black glass in the Roman Empire during the 1 st to 5 th centuries A.D. An Archaeological, Archaeometric and Historical approach", PhD Vrje Universiteit Brussel.

Cosyns P., Cagno S., Janssens K., Nys K., 2012, "The benefit of using chemical analysis in understanding archaeological glass. Case-study:
Roman black glass", in Proceedings of SPIE - The international Society for Optical Engineering, vol 8422, 842203-2, mis en ligne novembre 2012, DOI: 10.1117/12.975682, URL: https://www. researchgate.net/ publication/258717420.

Dahmen K., 2001, Untersuchungen zu Form und Funktion kleinformatiger Porträts der römischen Kaiserzeit, Scriptorium, Münster.

De Ridder A., 1913, Bronzes antiques du Louvre. I. Les figurines, Ernest Leroux, Paris.

Descamps S., 2007, "Augustus and Livia" dans Exp. Indianapolis, 2007, $n^{\circ} 33$, p. 93-94.

Descamps S., 2008, « Auguste et Livie » dans Exp. Arles, 2008, no 33a et b, p. 108-111.

Dran J.-C., Salomon J., Calligaro Th., Walter Ph., 2004, "Ion beam analysis of art works: 14 years of use in the Louvre", Nuclear Instruments and Methods in Physics Research, Section B7: Beam Interactions with Materials and Atoms, 219-220, p. 7-15.

Exp. Arles, 2008 : De l'esclave à l'empereur. L'art romain dans les collections du musée du Louvre [Exposition, Arles, musée départemental Arles antique, 20 décembre 2008-3 mai 2009, Giroire C., Roger D. (éds.)].

Exp. Indianapolis, 2007 : Roman Art from the Louvre [Exposition, Indianapolis, Indianapolis Museum of Art, September 23, 2007-January 6, 2008 ; Seattle, Seattle Art Museum, February 21-May 18, 2008 ; Oklahoma City, Oklahoma City Museum of Art, June 26-October 12, 2008, Giroire C., Roger D. (éds.)].

Exp. Paris, 2002 : Caylus mécène du roi, collectionneur. Les antiquités au XVIII ${ }^{e}$ siècle [Exposition, Paris, musée des Monnaies, médailles et antiques de la Bibliothèque nationale de France, 17 décembre 2002 au 17 mars 2003, Aghion I., Avisseau-Broustet M. (éds.)].

Exp. Paris, 2014 : Moi, Auguste, empereur de Rome... [Exposition, Paris, Grand Palais, Rome, Galeries nationales, 19 mars-13 juillet 2014, Giroire C., Roger D. (éds.)].

Fishwick D., Imperial Cult in the latin West: Studies in the Ruler Cult of the Western Provinces of the Roman Empire, II.1, Leiden-New York.

Freestone I. C., Stapleton C. P., 2015 , "Composition, technology and production of coloured glassed from the Roman mosaic vessels", in Bayley J., Freestone I. C., Jackson C. (dir.), Glass of the Roman world, Oxbow Books, Oxford \& Philadelphia, p. 61-76.

Formigli E., 2015, "The restoration of the Early Hellenistic Shipka Bronze Head", Archeologica Bulgarica XIX, 3, p. 1-22.

Frœhner W., 1873, « Auguste et Livie. Bustes en bronze - musée du Louvre ", Les Musées de France. Recueil des Monuments antiques, Paris, p.1-12.

Gedzeviciute V., Welter N., Schüssler U., Weiss C., 2009, "Chemical composition and colouring agents of Roman mosaic and millefiori glass, studied by electron microprobe analysis and Raman microspectroscopy", Archaeological Anthropological Science, 1, p. 15-29.

Héron de Villefosse A., 1907, Institut de France. Inauguration du buste de Prosper Mérimée à Cannes, le dimanche 28 auril 1907. Discours, Firmin-Didot et $\mathrm{C}^{\mathrm{ie}}$, imprimeurs de l'Institut de France, Paris.

Kaufmann-Heinimann A., 2004, « Götter im Keller, im Schiff und auf dem Berg. Alte und neue Statuettenfunde aus dem Imperium Romanum ", in Muşețeanu C. (dir.), The Antique Bronzes: Typology, Chronology, Authenticity, The Acta of the 16th International Congress of Antique Bronzes, Bucharest, May $26^{t h}-31^{s t}$, 2003, Bucharest, Editura Cetatea De Scaun., p. 249- 263.

Kersauson (de) K., 1986, Catalogue des portraits romains. I. Portraits de la République et d'époque julio-claudienne, RMN, Paris.

Lahusen G., Formigli, E., 2001, Römische Bildnisse aus Bronze: Kunst und Technik, Hirmer Verlag München, Munich.

Regert M., Colinart S., Degrand L., Decavallas O., 2001, "Chemical alteration and use of beeswax through time: accelerated ageing tests and analysis of archaeological samples from various environmental contexts", Archaeometry, 43 (4) p. 549-569.

Regert M., Langlois J., Colinart S., 2005, "Characterisation of wax works of art by gas chromatographic procedures", Journal of chromatography A, 1091, p. 124136.

Risser E., Saunders D., 2015, “The Bronze Apollo and Diana from Pompeii. An Example of Serial Production", Antike Kunst, Vol. 58, p. 78-96.

Robbiola L., Hurtel L.-P., 1995, "Standard Nature of the Passive Layers of Buried Archaeological Bronze. The Example of Two Roman Half-length Portraits", in MacLeod I. D., Pennec S. L., Robbiola L., Metal 95, Proceedings or the International Conference on Metals Conservation, Semur-en-Auxois, 25-28 septembre 1995, p. 109-117.

Rolley C., 1979, « Les bronzes antiques : objets d'art ou documents historiques ? ", dans Bérard C., Ducrey P. (éds.), Bronzes hellénistiques et romains. Tradition et renouveau. Actes du V colloque international sur les bronzes antiques, Lausanne, 8-13 mai 1978 (Cahiers d'archéologie romande $\mathrm{n}^{\circ} 17$ ), Lausanne.

Szewczyk M., 2014, « Auguste et Livie de Neuilly-le-Réal », dans Exp. Paris, 2014, $\mathrm{n}^{\text {os }} 71-72$, p. 115.

\section{Document inédit}

Letterlé F., 2004, Lettre datée du 26 mai 2004 dans le dossier des œuvres (musée du Louvre, département des Antiquités grecques, étrusques et romaines, Br 28-29). 